\title{
Perotrochus caledonicus (Gastropoda: Pleurotomariidae) revisited: descriptions of new species from the South-West Pacific
}

\author{
Patrick ANSEEUW ${ }^{1}$, Nicolas PUILLANDRE2,*, José UTGE², Philippe BOUCHET ${ }^{4}$ \\ ${ }^{1}$ Mispelstraat 18, 9820 Merelbeke, Belgium. \\ ${ }^{2}$ Institut de Systématique, Evolution, Biodiversité ISYEB - UMR7205 - CNRS, MNHN, UPMC, \\ EPHE, Muséum National d'Histoire Naturelle, Sorbonne Universités, \\ 43 Rue Cuvier, F-75231 Paris, France. \\ ${ }^{3}$ UMS 2700, Muséum National d'Histoire Naturelle, Département Systématique et Evolution, \\ 43 Rue Cuvier, F-75231 Paris, France. \\ ${ }^{4}$ Institut de Systématique, Evolution, Biodiversité ISYEB - UMR7205 - CNRS, MNHN, UPMC, \\ EPHE, Muséum National d'Histoire Naturelle, Sorbonne Universités, \\ 55 Rue Buffon, F-75231 Paris, France. \\ *Corresponding author: puillandre@mnhn.fr \\ ${ }^{1}$ urn:1sid:zoobank.org:author:89470910-9E6E-4B84-91E4-9C10019DD7DD \\ ${ }^{2}$ urn:1sid:zoobank.org:author:00565F2A-C170-48A1-AAD9-16559C536E4F \\ ${ }^{3}$ urn:1sid:zoobank.org:author:3888EAFD-B518-4979-A2DF-4A7A1D4B7F89 \\ ${ }^{4}$ urn:Isid:zoobank.org:author:FC9098A4-8374-4A9A-AD34-475E3AAF963A
}

\begin{abstract}
Morphological (shell) and molecular examination of a large suite of specimens of pleurotomariids from around New Caledonia and the Coral Sea reveals the existence of four species in the complex of Perotrochus caledonicus: Perotrochus deforgesi Métivier, 1990 and P. pseudogranulosus sp. nov. live allopatrically on the plateaus and guyots of the Coral Sea; Perotrochus caledonicus Bouchet \& Métivier, 1982 and Perotrochus wareni sp. nov. live sympatrically - but essentially not syntopically on the slopes of New Caledonia, Norfolk Ridge and the Loyalty Ridge. All species live in the 300-500 $\mathrm{m}$ interval, and together form a significant component of the mollusc fauna living on hard bottoms in the SW Pacific, with individual dredge hauls containing up to 25 specimens of Perotrochus.
\end{abstract}

Keywords. Perotrochus wareni sp. nov., Perotrochus pseudogranulosus sp. nov., Coral Sea, New Caledonia, COI Barcode gene.

Anseeuw P., Puillandre N., Utge J. \& Bouchet P. 2015. Perotrochus caledonicus (Gastropoda: Pleurotomariidae) revisited: descriptions of new species from the South-West Pacific. European Journal of Taxonomy 134: 1-23. http://dx.doi.org/10.5852/ejt.2015.134

\section{Introduction}

Perotrochus caledonicus Bouchet \& Métivier, 1982 and P. tangaroanus Bouchet \& Métivier, 1982 were the first pleurotomariids described from the South Pacific, from New Caledonia and from the Lau Ridge between New Zealand and Fiji (Bouchet \& Métivier 1982), respectively. Since then, additional species have been documented from New Caledonia (Perotrochus deforgesi Métivier, 1990 and Bayerotrochus 
boucheti (Anseeuw \& Poppe, 2001)) and Tonga (Bayerotrochus poppei Anseeuw, 2003). Moreover, many populations at first sight referable to Perotrochus caledonicus were sampled around New Caledonia and in the Coral Sea.

The type material of $P$. caledonicus from off southern New Caledonia consists of specimens with a macroscopically non-pustulose shell, with rather smooth to weakly beaded spiral cords. Soon after its description, it appeared that a more granulose or pustulose morph also occurred around New Caledonia. However, as such material appeared very similar in terms of size, color and general profile, it was considered to represent just a form of $P$. caledonicus. The two forms were reported in print (Anseeuw 1990; Anseeuw \& Goto 1996) and referred to as the "smooth form" and "pustulose form" of P. caledonicus, respectively. Since then, dealers and shell collectors have maintained this distinction, using the expression "caledonicus pustulose form" or "caledonicus granulose form", with some even suggesting that the latter could warrant full recognition as a species (e.g., www.conchology.be).

New material from New Caledonia and the Coral Sea has now allowed revisiting this issue, using an integrative taxonomic approach combining DNA sequencing and shell characters of both juveniles and adults. DNA sequences provide independent characters to test whether the different forms of $P$. caledonicus correspond to divergent lineages or not. The congruence of the different sets of characters analyzed revealed that three species were hidden under the name $P$. caledonicus, two of which are now described as new. In addition, Perotrochus deforgesi was found to be molecularly closely related to this complex - which was not suspected from shell characters alone - and in this paper we will designate these small Perotrochus from the SW Pacific together as the "Perotrochus caledonicus complex".

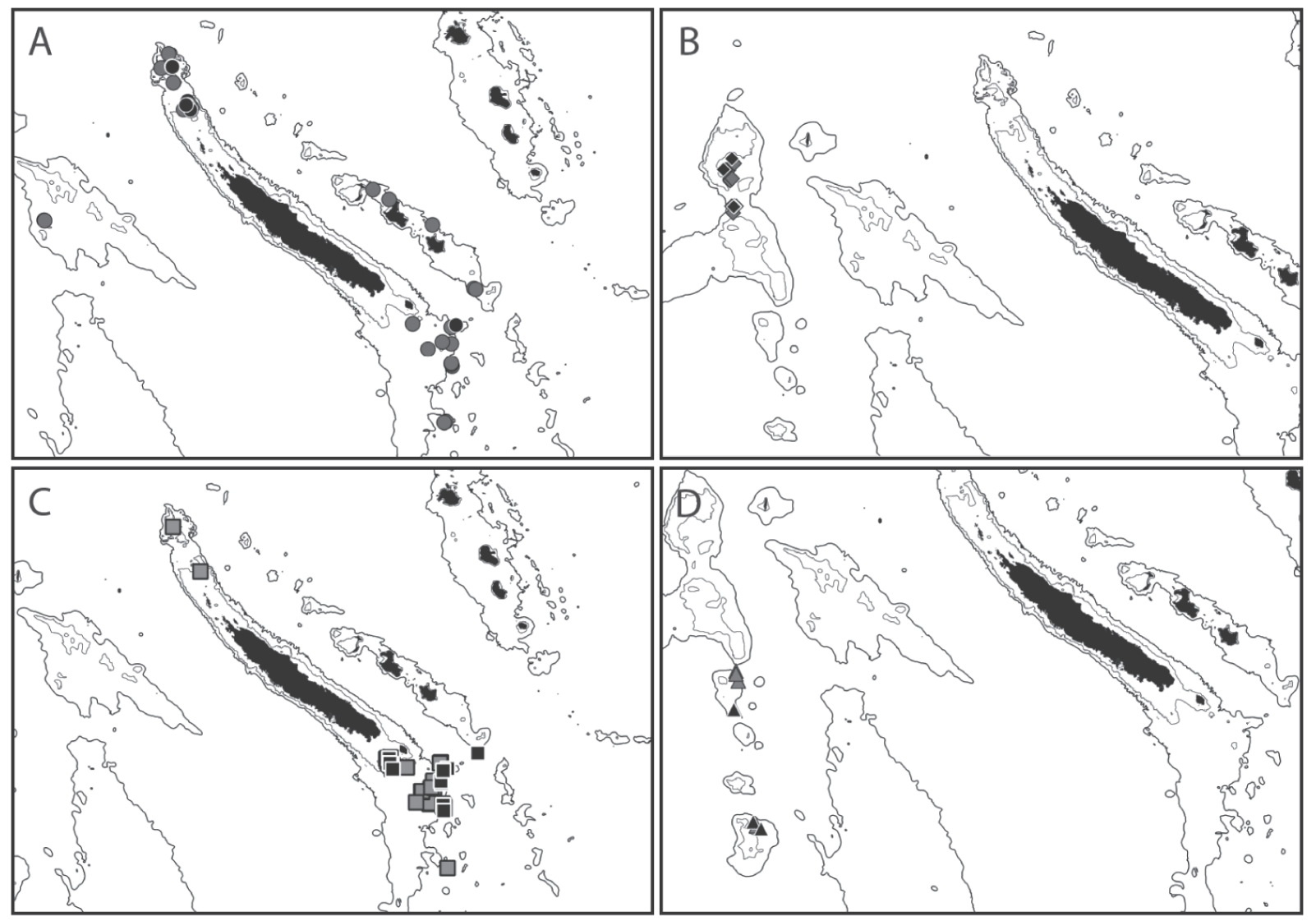

Fig. 1. Distribution maps of the four species. A. Perotrochus wareni sp. nov. B. Perotrochus deforgesi Métivier, 1990. C. Perotrochus caledonicus Bouchet \& Métivier, 1982. D. Perotrochus pseudogranulosus sp. nov. Stations with sequenced specimens in black. 


\section{Material and methods}

\section{Material}

A total of 284 lots and 879 specimens, including 59 sequenced specimens, attributable to the Perotrochus caledonicus complex were collected in the New Caledonia region, including the Coral Sea (Table 1, Fig. 1) during research cruises of the Tropical Deep-Sea Benthos program (Bouchet, Héros, Lozouet \& Maestrati 2008). After 2002, live-taken specimens were specifically processed on board for molecular analyses. A piece of the foot was cut and placed in $95^{\circ}$ ethanol; the remaining body and the shells were also preserved for further examination. All specimens are preserved at the Muséum National d'Histoire Naturelle (MNHN); each sequenced specimen is linked to a unique MNHN collection number. These specimens are registered in BOLD, the Barcode of Life database, and the corresponding sequences are also registered in GenBank (Table 1, Fig 1).

\section{DNA sequencing}

Total DNA was extracted from the piece of foot using the 6100 Nucleic Acid Prepstation system (Applied Biosystem) or the Epmotion 5075 robot (Eppendorf) following the manufacturer's recommendations. The barcode fragment of the COI gene $(658 \mathrm{bp})$ was tentatively amplified using the universal primers LCO1490 and HCO2198 (Folmer, Black, Hoeh, Lutz \& Vrijenhoek 1994). However, the rate of success was very low (less than 10\%), and several other protocols and primer pairs were tested. Finally, we were able to amplify most of the specimens, including several for each morphological species hypothesis, using the primers FISHR2 (Steinke \& Hanner 2011) and 140F (Ketmaier, Giusti \& Caccone 2006), and the following protocol: PCR reactions were performed in $20 \mu$ containing $3 \mathrm{ng}$ of DNA, $10 \times$ reaction buffer containing $15 \mathrm{mM} \mathrm{MgCl}{ }_{2}, 0.26 \mathrm{mM} \mathrm{dNTP}, 0.3 \mu \mathrm{M}$ of each primer, 5\% DMSO, $1 \mathrm{mg} / \mathrm{ml} \mathrm{BSA}$, and 1 unit of QBiotaq (MPBiomedicals). Amplification consisted of an initial denaturation step at $95^{\circ} \mathrm{C}$ for 5 ', followed by 38 cycles of denaturation at $95^{\circ} \mathrm{C}$ for $40^{\prime}$, annealing at $50^{\circ} \mathrm{C}$ for $40^{\prime}$, followed by extension at $72^{\circ} \mathrm{C}$ for $50^{\prime}$. The final extension was at $72^{\circ} \mathrm{C}$ for $3^{\prime}$. PCR products were purified and sequenced by the Eurofins sequencing facility. Both directions were sequenced to confirm accuracy of each sequence.

\section{Phylogenetic analyses}

In addition to the specimens of Perotrochus sequenced by us, COI sequences of Pleurotomariidae from GenBank were included in the dataset, as well as a sequence of Haliotis tuberculata (Haliotidae), used as outgroup (Table 1). Phylogenetic analyses were performed using MrBayes (Huelsenbeck, Ronquist \& Hall 2001), running two parallel analyses, consisting each of five Markov chains of 20,000,000 generations with a sampling frequency of one tree each 2,500 generations. The number of swaps was set to 3 , and the chain temperature at 0.02 . Parameters of the substitution model were estimated during the analysis (6 substitution categories, a gamma-distributed rate variation across sites approximated in four discrete categories and a proportion of invariable sites). A different model of substitution was applied for each codon position of the COI gene. Convergence of each analysis was evaluated using Tracer 1.4.1 (Rambaut \& Drummond 2007) to check that ESS values were all greater than 200 (default burning). K2P genetic distances were calculated with MEGA 5 (Tamura et al. 2011).

\section{Results}

\section{Morphological analyses}

With the exception of some juveniles, we could separate all the specimens of the Perotrochus caledonicus complex into four different morphotypes (Table 2): one group presents shell characters consistent with the name-bearing holotype of P. caledonicus as described by Bouchet \& Métivier (1982); the second group corresponds to the "rugose" or "pustulose" form, and is described below as $P$. wareni sp. nov.; a third group, with a geographically limited distribution within the Coral Sea, shows a microgranular 
sculpture and is described as $P$. pseudogranulosus sp. nov.; the fourth group is readily assignable to $P$. deforgesi.

\section{Phylogenetic analyses}

The phylogenetic analyses show that the $P$. caledonicus group is a well-supported clade (Posterior Probability PP =1) within the Pleurotomariidae (Fig. 2). Although based on a single gene, the tree would also suggest that the genus Perotrochus is not monophyletic. Furthermore, some GenBank sequences appear to be either misidentified or contaminated; for example, the sequence L78912.1, identified as Bayerotrochus teramachii (Kuroda, 1955), is almost identical to three sequences identified as B. midas (Bayer, 1965). Within P. caledonicus s.l., the analysis of the COI gene diversity revealed the presence of four groups, each corresponding to a well supported clade in the phylogenetic tree ( $\mathrm{PP}=1$ for each of them), and totally congruent with the morphological analysis. $P$. deforgesi is the sister-clade of a group that includes the specimens attributed to $P$. caledonicus and $P$. wareni sp. nov. The mean K2P genetic distance between $P$. wareni sp. nov. and P. caledonicus is $3.8 \%$, which is similar to the distance

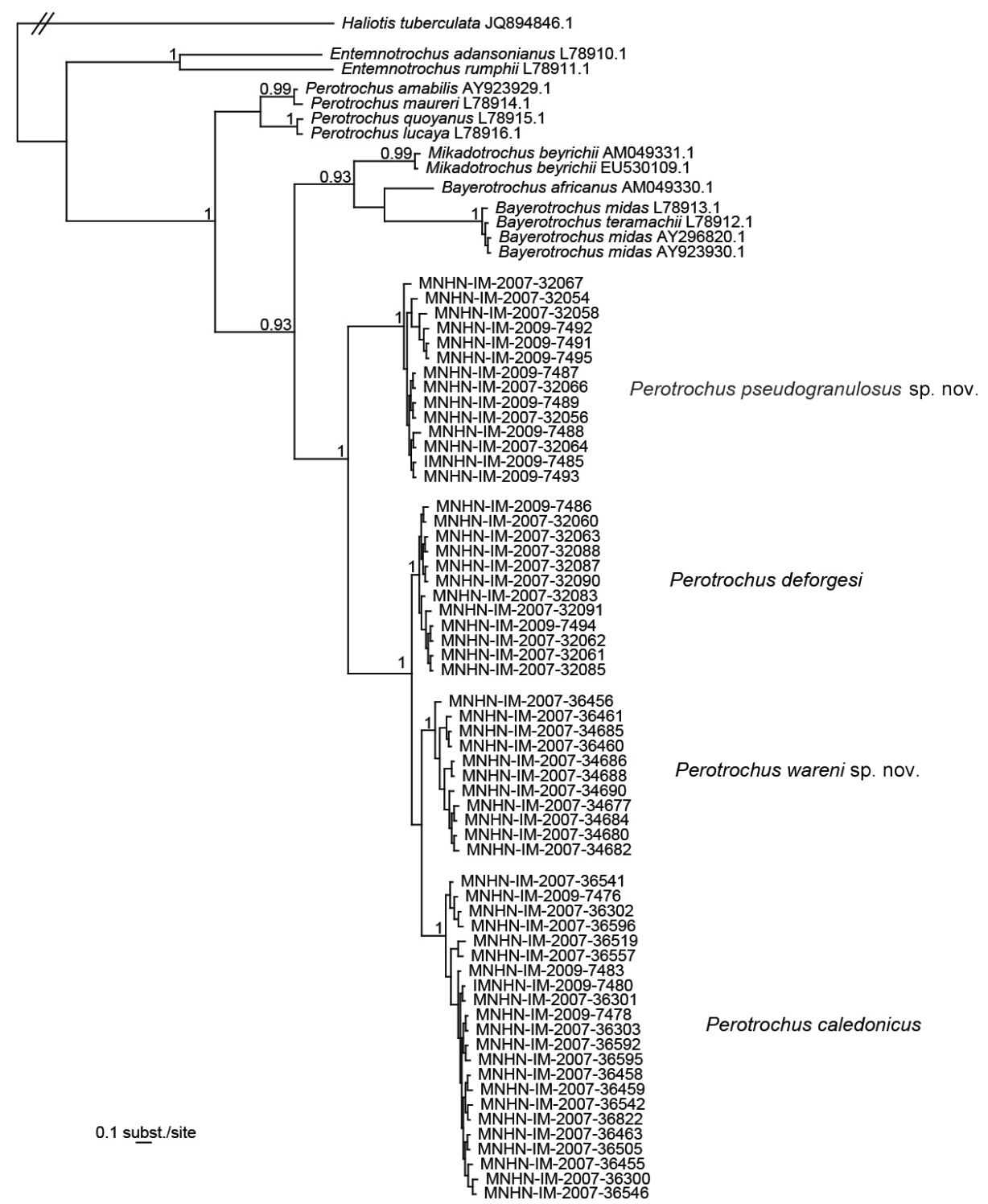

Fig. 2. Bayesian phylogenetic tree. Posterior probabilities $(>0.90)$ are shown for each node. 
between $P$. deforgesi and $P$. caledonicus (3.7\%) and greater than the distance between $P$. deforgesi and $P$. wareni sp. nov. (2.1\%). This would tend to confirm that if $P$. deforgesi is considered a valid species - which is morphologically indisputable - then the two morphs (P. caledonicus s.s. and P. wareni sp. nov.), included until now in $P$ caledonicus, should be ranked as species as well. More surprisingly, P. pseudogranulosus sp. nov. is the sister-group to a clade that includes the other three species, with a mean genetic distance of $9.9 \%$ to the other three species, confirming its validity as a separate species.

\section{Systematics}

Phylum Mollusca Cuvier, 1795

Class Gastropoda Cuvier, 1795

Subclass Vetigastropoda Salvini-Plawen, 1980

Superfamily Pleurotomarioidea Swainson, 1840

Family Pleurotomariidae Swainson, 1840

Genus Perotrochus P. Fischer, 1885

\section{Comparative material (all in MNHN)}

Perotrochus caledonicus. 135 lots comprising 560 specimens (Table 1).

Perotrochus deforgesi. 36 lots comprising 59 specimens (Table 1).

Perotrochus wareni sp. nov. urn:Isid:zoobank.org:act:51E7B304-66D2-4AE6-84C0-3A4427203065

Figs 1A, 3A-F, 4A-J

\section{Etymology}

This new species is named in honor of Dr Anders Warén of Naturhistoriska Riksmuseet, Stockholm, in recognition of his lifetime interest in deep-sea exploration and his participation in expeditions around New Caledonia and elsewhere in the South Pacific, many of which yielded specimens used in this paper.

\section{Material examined}

73 lots comprising 176 specimens (Table 1).

\section{Type material}

Holotype

NEW CALEDONIA: a sequenced specimen, MNHN-IM-2007-36460.

Paratypes

NEW CALEDONIA: MNHN-IM-2007-34680 (Fig. 4A-B); MNHN-IM-2007-34684 (Fig. 4C-D); MNHN-IM-2007-34685 (Fig. 4E-F); MNHN-IM-2007-36456 (Fig. 4G-H); MNHN-IM-2007-36461 (Fig. 4I-J).

\section{Type locality}

Norfolk Ridge, Munida Bank, 2259’ S, 168²1’ E, 320-390 m (TERRASSES sta. DW3101).

Description (holotype)

Shell of medium size, solid, thick, general profile rather conical, with weakly convex, rather straightsided whorls, with a diameter a little smaller than its height $(\mathrm{H} / \mathrm{D}$ ratio $=1.08)$, numbering 10.5 teleoconch whorls, with a mean spire angle of $65^{\circ}$ with weakly impressed suture, whorl surface dull. Protoconch glassy, rather obtusely depressed. Teleoconch with heavily beaded spiral cords very early on. Dominant 


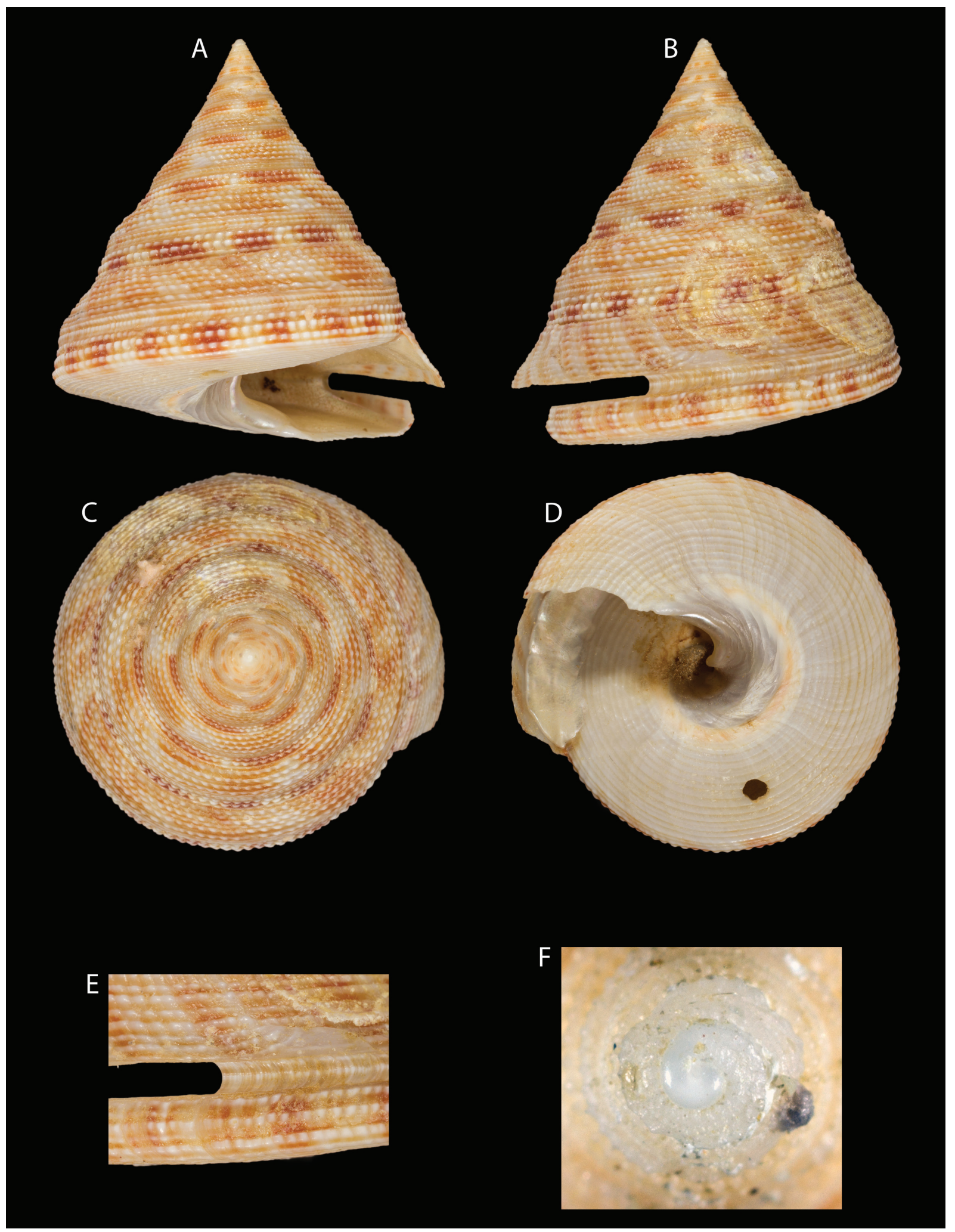

Fig. 3. Perotrochus wareni sp. nov., MNHN-IM-2007-36460, holotype $(\mathrm{H}=50.3 \mathrm{~mm})$. A-D. Teleoconch. E. Slit. F. Protoconch. 
ANSEEUW P. et al., New species of Pleurotomariidae

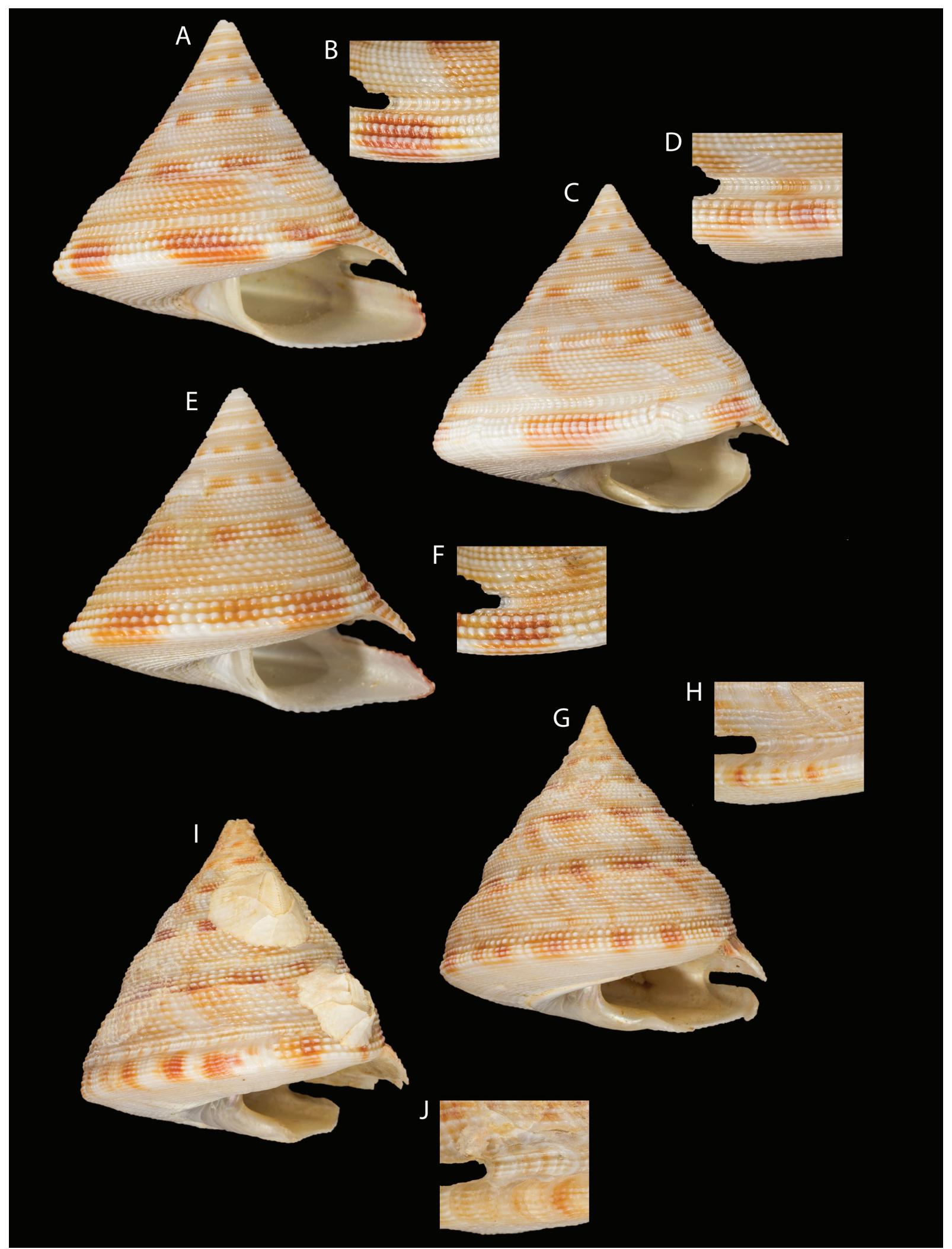

Fig. 4. Perotrochus wareni sp. nov., paratypes. A-B. MNHN-IM-2007-34680 (H = $28.2 \mathrm{~mm})$. C-D. MNHN-IM-2007-34684 (H = $43.8 \mathrm{~mm})$. E-F. MNHN-IM-2007-34685 ( $\mathrm{H}=21.5 \mathrm{~mm})$. G-H. MNHN-IM-2007-36456 ( $\mathrm{H}=55.1 \mathrm{~mm})$. I-J. MNHN-IM-2007-36461 $(\mathrm{H}=48.8 \mathrm{~mm})$. 
teleoconch sculpture consisting of finely but strongly beaded spiral cords, intersecting less marked axial riblets. Periphery of basal disc crenulated due to a strongly marked spiral cord running at edge of disc. On last whorl, 13 spiral cords above selenizone, 4 spiral cords below, and 2 major cords in the selenizone itself. Slit short, about $17^{\text {th }}$ the circumference of last whorl, situated below midwhorl, and very narrow. Aperture rectangularly depressed. Basal disc rather flat, depressed in its center and sharply edged at its periphery, with a very wide (extending over $40 \%$ of base diameter) callus pad which is finely ridged radially. Inside aperture, inner slit lips only partially covered by nacre, leaving a V-shaped area uncovered (approximately $25 \%$ of surface of inner slit lip extremity in aperture uncovered by nacre, showing only porcellaneous layer). Nacre coverage thick, no surface sculpture showing through it. Background color yellowish beige, with some faint axial orange-red flammulations, not really arranged into a distinct checker-board pattern; basal disc of same colour, with some faint orange axial flammulation reaching only to basal disc edge. Operculum small, multispiral, circular, light yellowish.

\section{Measurements}

Maximum basal diameter (D) $46.75 \mathrm{~mm}$, minimum diameter $43.9 \mathrm{~mm}$. Height (H) $50.27 \mathrm{~mm}$. H/D = 1.08. Length of slit at upper margin $26.7 \mathrm{~mm}$, at lower margin $16.2 \mathrm{~mm}$. Slit width: $1.6 \mathrm{~mm}$; slit length: $1 / 6.51^{\text {th }}$ of circumference of last whorl. Weight of empty shell $26.3 \mathrm{~g}$.

\section{Discussion}

One of the distinctive shell characters separating Perotrochus wareni sp. nov. from P. caledonicus is the well-marked beading on the teleoconch spiral cords, visible also on the earlier whorls, giving it at first glance its typical pustulose or granular appearance. The intensity of the beading varies between specimens, leading to "heavily beaded"/"very pustulose" specimens and to "weakly beaded" / "light pustulose" specimens, with all different intergrades. This variability may reflect environmental conditions, as the beading intensity is generally stable within one haul / lot and varies between hauls / lots. Leaving aside beading intensity, shell characters are quite stable in $P$. wareni sp. nov. The general profile of the teleoconch, the outline of the aperture, the selenizone and slit width, and the extension of the callus on the basal disc, all show consistent differences between $P$. wareni sp. nov., P. caledonicus and P. pseudogranulosus sp. nov. (Table 2). Perotrochus wareni sp. nov. also bears some resemblance to P. gotoi Anseeuw, 1990, and, in fact, a specimen of the granulated $P$. "cfr. caledonicus" ([i.e., $P$. wareni sp. nov.) had been used for comparison at the time of its original description (Anseeuw 1990). Perotrochus wareni sp. nov. can be separated from P. gotoi by its somewhat shorter slit length $\left(1 / 6.51^{\text {th }}\right.$ of basal diameter in wareni sp. nov. vs $1^{1 / 6}$ in gotoi), the larger number of spiral cords on adult specimens, the more irregularly banded checkerboard colour pattern in the area below the selenizone, the heavier and thicker shell (around 60-70\% heavier at comparable shell sizes), the less extensive area uncovered by the nacreous layer inside the apertural inner slit lip extremities, and the umbilical callus occupying a much larger surface on the basal disc ( $45 \%$ in $P$. wareni sp. nov. vs $28 \%$ in $P$. gotoi). The two species, however, share (also with Mikadotrochus salmianus (Rolle, 1899) the nacreous coverage of the inner slit lips in the aperture, a feature that separates them from Perotrochus caledonicus s.s. and P. pseudogranulosus sp. nov. (Table 2, Figs 3-4, 7). Other features, like a deeper, more intense colour pattern on the teleoconch and basal disc, fine microgranulosity on the spiral cords, a thin, light shell, and a more lustrous shell surface, further separate Perotrochus pseudogranulosus sp. nov. from P. wareni sp. nov. (Table 2, Figs 3-6). 
ANSEEUW P. et al., New species of Pleurotomariidae

\section{Perotrochus pseudogranulosus sp. nov. urn:1sid:zoobank.org:act:CE972B51-FB50-4C06-B1B2-DD01A6BC737D}

Figs 1D, 5A-F, 6A-J

\section{Etymology}

The specific epithet emphasizes the beaded spiral sculpture of the species.

\section{Material examined}

40 lots comprising 84 specimens (Table 1).

\section{Type material}

Holotype

NEW CALEDONIA: a sequenced specimen, MNHN-IM-2009-7495.

Paratypes

NEW CALEDONIA: MNHN-IM-2007-32058 (Fig. 6A-B); MNHN-IM-2007-32066 (Fig. 6C-D); MNHN-IM-2009-7485 (Fig. 6E-F); MNHN-IM-2009-7491 (Fig. 6G-H); MNHN-IM-2009-7493 (Fig. 6I-J).

\section{Type locality}

Coral Sea, Capel Bank, 2445’ S, 15942’ E, 348-354 m (EBISCO sta. CP2494).

\section{Description (holotype)}

Shell of medium size, light, thin, general profile rather conical, with weakly convex to straight-sided whorls with weakly impressed suture, diameter significantly exceeding height $(\mathrm{H} / \mathrm{D}=0.78)$, numbering 8 teleoconch whorls, with a mean spire angle of $80^{\circ}$. Protoconch ivory white, depressed. Dominant sculpture of teleoconch consisting of numerous lightly beaded spiral cords, with microsculptural pattern of fine radiating threads, giving the entire whorl surface a shiny metallic luster. On last whorl, 11 spiral cords above selenizone, 7 below and 3 major cords in the selenizone itself. Slit long, about 1/5 the circumference of the last whorl, situated below midwhorl, and rather narrow. Aperture depressed. Basal disc rather flattened, with angular edge, with a relatively narrow (extending over $30 \%$ of base diameter) light nacreous callus pad which is finely ridged radially and ends in a raised porcellaneous edge. Inside the aperture inner slit lips nearly completely covered by nacre, leaving a narrow area (approximately $15 \%$ of the surface) parallel to the inner slit lips uncovered, showing only porcellaneous layer. Background colour yellowish beige, with intense reddish crimson colour markings arranged in very regular checkerboard pattern, overall reinforcing color intensity; basal disc showing some contrasting reddish crimson flammulations, particularly visible at its periphery, and more yellowish tan towards the center. Operculum small, multispiral, circular, light yellowish (fallen off/missing in holotype).

\section{Measurements}

Maximum basal diameter (D) $64.9 \mathrm{~mm}$, minimum diameter $59.1 \mathrm{~mm}$. Height $(\mathrm{H}) 50.7 \mathrm{~mm}$. H/D = 0.78 . Depth of slit at upper margin $42.1 \mathrm{~mm}$, depth of slit at lower margin $26.9 \mathrm{~mm}$. Slit width $3.1 \mathrm{~mm}$. Slit length: $1 / 5.64^{\text {th }}$ of circumference of last whorl. Weight of empty shell $42.6 \mathrm{~g}$.

\section{Discussion}

Perotrochus pseudogranulosus sp. nov. most closely resembles P. caledonicus (Fig. 7) at first glance, but is distinguished by its more conical and higher shell, a more flattened basal disc profile and a more intense and regular checkerboard colour pattern and more lustrous shell surface. It differs from $P$. wareni sp. nov. by its weakly beaded spiral cords with a microsculpture of fine radiating threads, its more intensely 


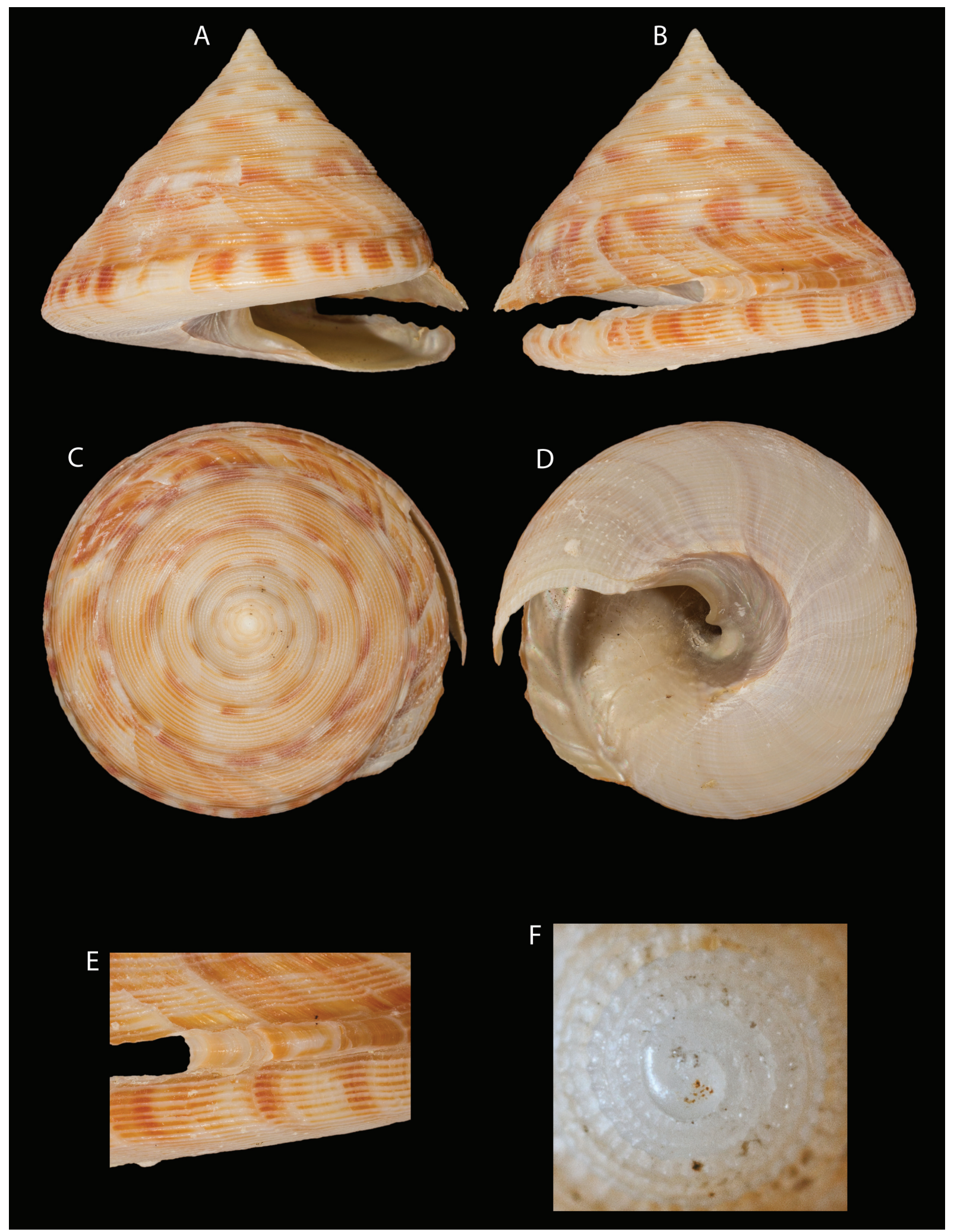

Fig. 5. Perotrochus pseudogranulosus sp. nov., MNHN-IM-2009-7495, holotype $(\mathrm{H}=54.3 \mathrm{~mm})$. A-D. Teleoconch. E. Slit. F. Protoconch. 
ANSEEUW P. et al., New species of Pleurotomariidae

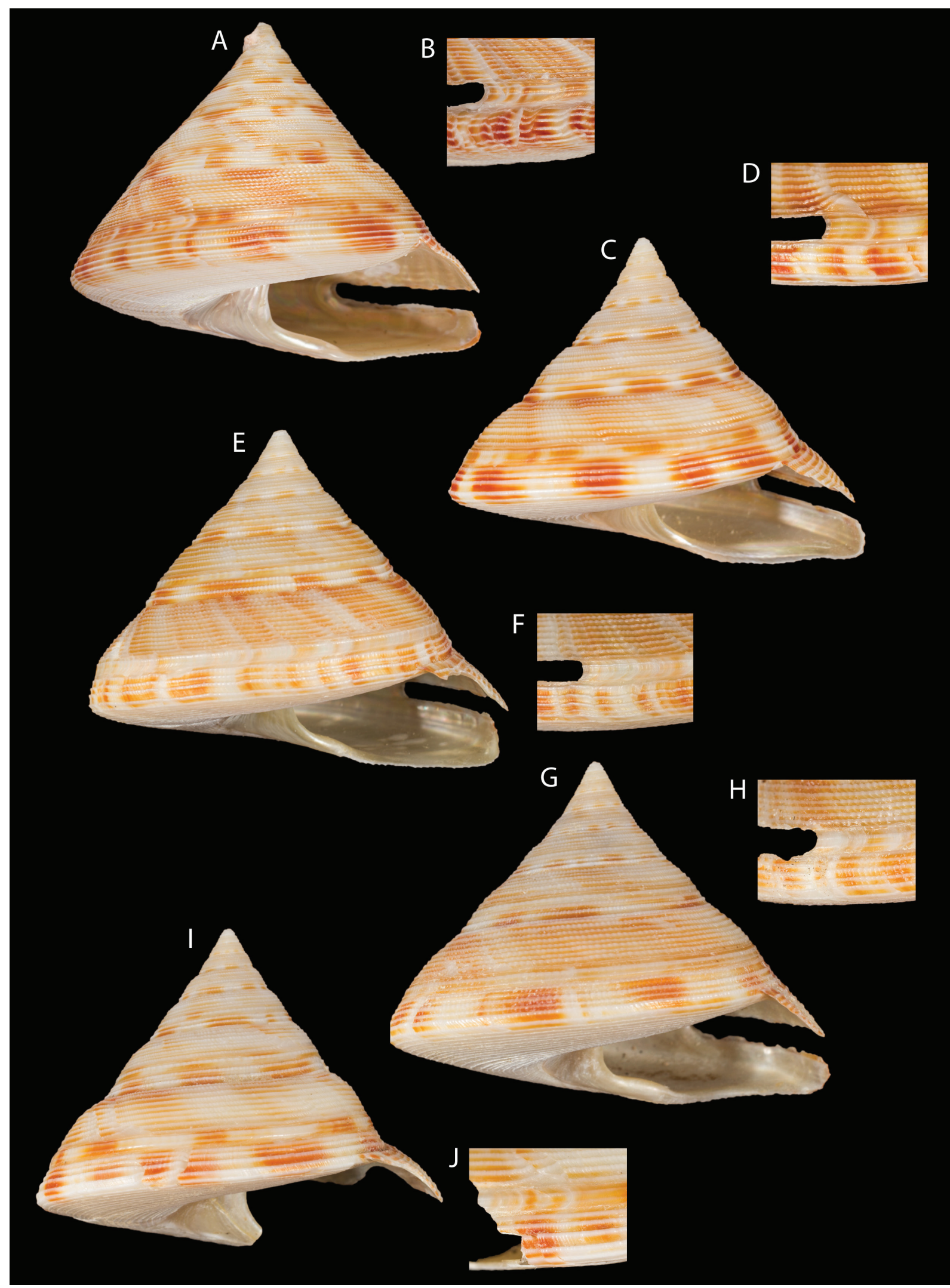

Fig. 6. Perotrochus pseudogranulosus sp. nov., paratypes. A-B. MNHN-IM-2007-32058 (H = $53 \mathrm{~mm})$. C-D. MNHN-IM-2007-32066 $(\mathrm{H}=29.8 \mathrm{~mm})$. E-F. MNHN-IM-2009-7485 $(\mathrm{H}=42.1 \mathrm{~mm})$. G-H. MNHN-IM-2009-7491 (H = 36 mm). I-J. MNHN-IM-2009-7493 $(\mathrm{H}=32.1 \mathrm{~mm})$. 
marked checkerboard colour pattern, with metallic luster, a much longer slit and a much smaller callus pad area on the basal disc. Finally, it differs from P. deforgesi (Fig. 7), the only other species occurring in the Coral Sea, by its general outline which is distinctly higher conical, its thin shell, its more intense checkerboard colour markings, its less granular spiral cords and smaller callus pad coverage on the basal disc.

Some specimens of $P$. pseudogranulosus sp. nov. have over the years turned up in the shell trade as "P. cfr. caledonicus", supposedly originating from NW Australia or even from the South China Sea (Anseeuw \& Goto 1996). However, based on the lack of precise and trustworthy locality data, the lack of more recent confirmation of those alleged findings and, most of all, the general unavailability of such material for study, we reject these localities as intentionally or unintentionally unproven and unverifiable.

\section{Discussion}

The geographical distribution within the New Caledonia region (Fig. 1) is different for the four species. Many samples of Perotrochus caledonicus s.s. originate from the SW off Ile des Pins, southern New Caledonia, with scattered specimens from Norfolk Ridge, the Loyalty Ridge and the Far North of New Caledonia (Grand Passage); the average depth of occurrence, based on 135 lots, is 407 meters. The distribution of Perotrochus wareni sp. nov. includes Norfolk Ridge, the Loyalty Ridge, the Far North of New Caledonia (Grand Passage) and the Lansdowne Plateau in the Coral Sea; the average depth of occurrence, based on 73 lots, is 344 meters. Perotrochus caledonicus and P. wareni sp. nov. thus co-occur locally in the south of New Caledonia, Norfolk Ridge and Grand Passage, where they have different bathymetric preferences, although they were sometimes found at the same stations (LITHIST sta. CP14 and sta. CP16; NORFOLK1 sta. DW1658 and sta. DW1709; MUSORSTOM4 sta. DW222; TERRASSES sta. DW3101 and sta. DW3110). Perotrochus pseudogranulosus sp. nov. has a distribution restricted to the Chesterfield Plateau, with a mean depth of 360 meters based on 40 lots. Perotrochus deforgesi is also restricted to the Coral Sea, but occurs only on the Bellona Plateau and Capel Bank, thus allopatrically with regard to P. pseudogranulosus sp. nov., but at similar depths (mean depth of 380 meters based on 36 lots).

The morphological, molecular and microdistribution data, thus all converge to support the conclusion that there are four small Perotrochus in the New Caledonia region, of which two have been described as new in the present paper.

At genus level, although Anseeuw \& Poppe (2005) and Aktipis \& Giribet (2010) had used the combinations Mikadotrochus caledonicus and M. deforgesi, the molecular phylogeny shows that Perotrochus caledonicus and P. deforgesi are not congeneric with Pleurotomaria beyrichii Hilgendorf, 1877, the type species of Mikadotrochus Lindholm, 1927. However, the tree also shows that the four small New Caledonia Perotrochus do not form a monophyletic group with Pleurotomaria quoyana P. Fischer \& Bernardi, 1856, the type species of Perotrochus P. Fischer, 1885. Perotrochus quoyanus is a species from the western Atlantic, and the species (P. amabilis (Bayer, 1963), P. maureri Harasewych \& Askew, 1993, P. lucaya Bayer, 1965) that cluster with it in the tree are also from the western Atlantic. It thus appears that, although they are traditionally placed in Perotrochus (e.g., Anseeuw \& Goto 1996; Harasewych 2002), the small pleurotomariids from the Pacific are not congeneric with true Perotrochus from the western Atlantic, and a new genus will have to be established to classify them.

\section{Acknowledgments}

The material on which this paper is based has been accumulated during many expeditions of the Tropical Deep-Sea Benthos programme in the New Caledonia region. We refer to Bouchet et al. (2008) for an 


\section{ANSEEUW P. et al., New species of Pleurotomariidae}

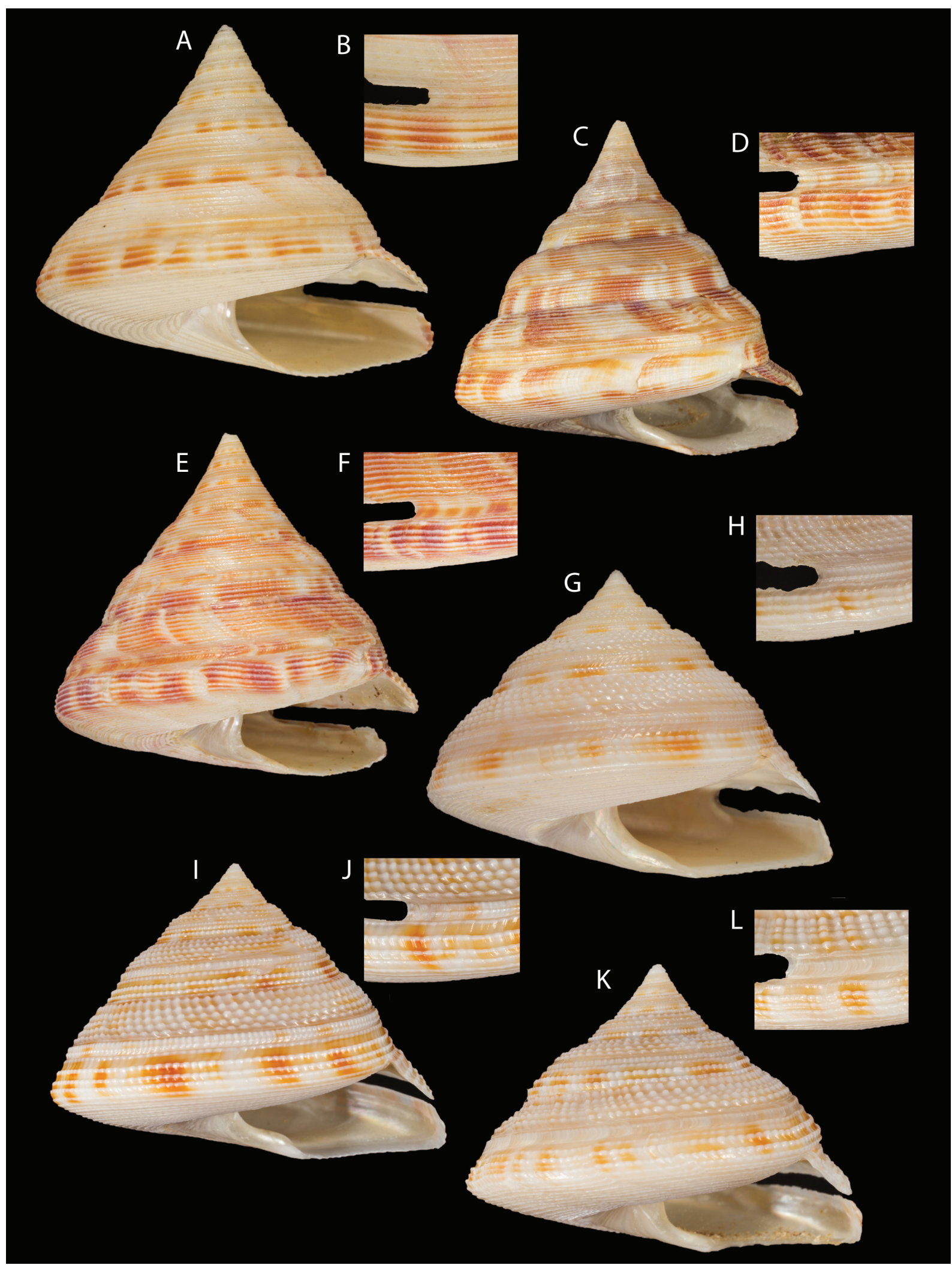

Fig. 7. A-F. Perotrochus caledonicus Bouchet \& Métivier, 1982. A-B. MNHN-IM-2000-1262, holotype $(\mathrm{H}=31.2 \mathrm{~mm})$. C-D. MNHN-IM-2007-36300 (H= 51.2 mm). E-F. MNHN-IM-2007-36301 ( $\mathrm{H}=47.5$ $\mathrm{mm})$. - G-L. Perotrochus deforgesi Métivier, 1990. G-H. MNHN-IM-2000-1391, holotype (H = 33.9 mm). I-J. MNHN-IM-2007-32062 ( $\mathrm{H}=33.7$ mm). K-L. MNHN-IM-2007-32085 (H=25.1 mm). 
overview of the programme and the acknowledgements to the captains, principal scientists and crew involved are applicable here. We thank Yoshihiro Goto and Guido Poppe, with whom we discussed the taxonomic hypotheses presented in this paper. Manuel Tenorio and Javier Conde attempted a morphometric analysis (results not shown). Barbara Buge, Virginie Héros, Julien Brisset and Philippe Maestrati curated the material, Philippe Maestrati and Manuel Caballer-Gutiérrez provided the photos. This work was supported by the Service de Systématique Moléculaire (UMS 2700 CNRS-MNHN), and we acknowledge the advice of M.G. Harasewych on DNA amplification in pleurotomariids.

\section{References}

Aktipis S.W. \& Giribet G. 2010. A phylogeny of Vetigastropoda and other "archaeogastropods": reorganizing old gastropod clades. Invertebrate Biology 129 (3): 220-240. http://dx.doi.org/10.1111/ j.1744-7410.2010.00198.x

Anseeuw P. 1990. A new species of pleurotomariid gastropod from the Philippines. La Conchiglia 22: $10-15$.

Anseeuw P. \& Goto Y. 1996. The Living Pleurotomariidae: 1. Elle Scientific Publications, Osaka.

Anseeuw P. \& Poppe G. 2005. Pleurotomariidae: An iconographic visit anno 2005. Visaya supplement 1, Cebu, Philippines.

Bouchet P., Héros V., Lozouet P. \& Maestrati P. 2008. A quarter-century of deep-sea malacological exploration in the South and West Pacific: Where do we stand? How far to go? In: Héros V., Cowie R.H. \& Bouchet P. (eds) Tropical Deep-Sea Benthos 25. Mémoires du Muséum national d'Histoire naturelle 196: 9-40. Muséum national d'Histoire naturelle, Paris.

Bouchet P. \& Métivier B. 1982. Living Pleurotomariidae (Mollusca: Gastropoda) from the South Pacific. New Zealand Journal of Zoology 9: 309-317.

Folmer O., Black M., Hoeh W., Lutz R. \& Vrijenhoek R. 1994. DNA primers for amplification of mitochondrial cytochrome c oxidase subunit I from diverse metazoan invertebrates. Molecular Marine Biology and Biotechnology 3: 294-299.

Harasewych M.G. 2002. Pleurotomarioidean gastropods. Advances in Marine Biology 42: 235-292.

Huelsenbeck J.P., Ronquist F. \& Hall B. 2001. MrBayes: Bayesian inference of phylogeny. Bioinformatics 17: 754-755. http://dx.doi.org/10.1093/bioinformatics/17.8.754

Ketmaier V., Giusti F. \& Caccone A. 2006. Molecular phylogeny and historical biogeography of the land snail genus Solatopupa (Pulmonata) in the peri-Tyrrhenian area. Molecular Phylogenetics and Evolution 39 (2): 439-451. http://dx.doi.org/10.1016/j.ympev.2005.12.008

Métivier B. 1990 Description of a new Perotrochus from the Coral Sea, Southwest Pacific (Gastropoda: Pleurotomariidae). Venus 49 (1): 1-7.

Rambaut A. \& Drummond A.J. 2007. Tracer v. 1.4. Available from http://beast.bio.ed.ac.uk/Tracer [accessed on 25 Jun. 2014].

Steinke D. \& Hanner R. 2011. The FISH-BOL collaborators' protocol. Mitochondrial DNA 22 (S1): 10-14. http://dx.doi.org/10.3109/19401736.2010.536538

Tamura K., Peterson D., Peterson N., Stecher G., Nei M. \& Kumar S. 2011. MEGA5: Molecular Evolutionary Genetics Analysis using Maximum Likelihood, Evolutionary Distance, and Maximum Parsimony methods. Molecular Biology and Evolution 28: 2731-2739. http://dx.doi.org/10.1093/ $\underline{\text { molbev/msr121 }}$ 
Manuscript received: 1 April 2015

Manuscript accepted: 11 June 2015

Published on: 24 August 2015

Topic editor: Rudy Jocqué

Desk editor: Kristiaan Hoedemakers

Printed versions of all papers are also deposited in the libraries of the institutes that are members of the EJT consortium: Muséum National d'Histoire Naturelle, Paris, France; Botanic Garden Meise, Belgium; Royal Museum for Central Africa, Tervuren, Belgium; Natural History Museum, London, United Kingdom; Royal Belgian Institute of Natural Sciences, Brussels, Belgium; Natural History Museum of Denmark, Copenhagen, Denmark. 
Table 1. List of specimens.

\begin{tabular}{|c|c|c|c|c|c|c|c|c|}
\hline MNHN ID & $\mathbf{N}$ & SPECIES & MNHN EXPEDITION & STATION & GENERAL AREA & COORDINATES; DEPTH (m) & BOLD ID & GENBANK \# \\
\hline IM-2000-1262 & 1 & P. caledonicus & VAUBAN & DR15 & & $22^{\circ} 49^{\prime} \mathrm{S}, 167^{\circ} 12^{\prime} \mathrm{E} ; 390-395$ & & \\
\hline IM-2000-1324 & 2 & P. deforgesi & CHALCAL 1 & DC32 & Chesterfield & $19^{\circ} 43^{\prime} \mathrm{S}, 158^{\circ} 33^{\prime} \mathrm{E} ; 350$ & & \\
\hline IM-2000-1361 & 1 & P. deforgesi & MUSORSTOM 5 & DW337 & Chesterfield & $19^{\circ} 54^{\prime} \mathrm{S}, 158^{\circ} 38^{\prime} \mathrm{E} ; 412-430$ & & \\
\hline IM-2000-1387 & 2 & P. caledonicus & VAUBAN & DR15 & & $22^{\circ} 49^{\prime} \mathrm{S}, 167^{\circ} 12^{\prime} \mathrm{E} ; 390-395$ & & \\
\hline IM-2000-1389 & 1 & P. deforgesi & MUSORSTOM 5 & DC361 & Chesterfield & $19^{\circ} 52^{\prime} \mathrm{S}, 158^{\circ} 38^{\prime} \mathrm{E} ; 400$ & & \\
\hline IM-2007-32054 & 1 & P. pseudogranulosus & EBISCO & DW2530 & S Nova Bank & $22^{\circ} 48^{\prime} \mathrm{S}, 159^{\circ} 23^{\prime} \mathrm{E} ; 338-343$ & PLERO010-15 & KR087206 \\
\hline IM-2007-32055 & 1 & P. pseudogranulosus & EBISCO & DW2530 & S Nova Bank & $22^{\circ} 48^{\prime} \mathrm{S}, 159^{\circ} 23^{\prime} \mathrm{E} ; 338-343$ & & \\
\hline IM-2007-32056 & 1 & P. pseudogranulosus & EBISCO & DW2530 & S Nova Bank & $22^{\circ} 48^{\prime} \mathrm{S}, 159^{\circ} 23^{\prime} \mathrm{E} ; 338-343$ & PLERO011-15 & KR087205 \\
\hline IM-2007-32057 & 1 & P. pseudogranulosus & EBISCO & DW2530 & S Nova Bank & $22^{\circ} 48^{\prime} \mathrm{S}, 159^{\circ} 23^{\prime} \mathrm{E} ; 338-343$ & & \\
\hline IM-2007-32058 & 1 & P. pseudogranulosus & EBISCO & DW2501 & Capel Bank & $24^{\circ} 50^{\prime} \mathrm{S}, 159^{\circ} 51^{\prime} \mathrm{E} ; 325-520$ & PLERO058-15 & KR087194 \\
\hline IM-2007-32059 & 1 & P. wareni & EBISCO & DW2631 & S Lansdowne & $21^{\circ} 03^{\prime} \mathrm{S}, 160^{\circ} 44^{\prime} \mathrm{E} ; 372-404$ & & \\
\hline IM-2007-32060 & 1 & P. deforgesi & EBISCO & DW2577 & N Bellona & $20^{\circ} 20^{\prime} \mathrm{S}, 158^{\circ} 39^{\prime} \mathrm{E} ; 399-602$ & PLERO012-15 & KR087218 \\
\hline IM-2007-32061 & 1 & P. deforgesi & EBISCO & DW2577 & N Bellona & $20^{\circ} 20^{\prime} \mathrm{S}, 158^{\circ} 39^{\prime} \mathrm{E} ; 399-602$ & PLERO002-15 & KR087208 \\
\hline IM-2007-32062 & 1 & P. deforgesi & EBISCO & CP2567 & NW Bellona & $20^{\circ} 20^{\prime} \mathrm{S}, 158^{\circ} 42^{\prime} \mathrm{E} ; 395-400$ & PLERO013-15 & KR087217 \\
\hline IM-2007-32063 & 1 & P. deforgesi & EBISCO & CР2593 & Chesterfield & $19^{\circ} 43^{\prime} \mathrm{S}, 158^{\circ} 32^{\prime} \mathrm{E} ; 300-323$ & PLERO014-15 & KR087216 \\
\hline IM-2007-32064 & 1 & P. pseudogranulosus & EBISCO & DW2526 & S Nova Bank & $22^{\circ} 46^{\prime} \mathrm{S}, 159^{\circ} 23^{\prime} \mathrm{E} ; 330-340$ & PLERO015-15 & KR087193 \\
\hline IM-2007-32065 & 1 & P. pseudogranulosus & EBISCO & DW2526 & S Nova Bank & $22^{\circ} 46^{\prime} \mathrm{S}, 159^{\circ} 23^{\prime} \mathrm{E} ; 330-340$ & & \\
\hline IM-2007-32066 & 1 & P. pseudogranulosus & EBISCO & DW2526 & S Nova Bank & $22^{\circ} 46^{\prime} \mathrm{S}, 159^{\circ} 23^{\prime} \mathrm{E} ; 330-340$ & PLERO016-15 & KR087204 \\
\hline IM-2007-32067 & 1 & P. pseudogranulosus & EBISCO & DW2525 & S Nova Bank & $22^{\circ} 48^{\prime} \mathrm{S}, 159^{\circ} 23^{\prime} \mathrm{E} ; 408-410$ & PLERO017-15 & KR087203 \\
\hline IM-2007-32068 & 1 & P. wareni & EBISCO & DW2634 & S Lansdowne & $21^{\circ} 05^{\prime} \mathrm{S}, 160^{\circ} 46^{\prime} \mathrm{E} ; 342-347$ & & \\
\hline IM-2007-32069 & 1 & P. wareni & EBISCO & DW2634 & S Lansdowne & $21^{\circ} 05^{\prime} \mathrm{S}, 160^{\circ} 46^{\prime} \mathrm{E} ; 342-347$ & & \\
\hline IM-2007-32070 & 1 & P. pseudogranulosus & EBISCO & DW2525 & S Nova Bank & $22^{\circ} 48^{\prime} \mathrm{S}, 159^{\circ} 23^{\prime} \mathrm{E} ; 408-410$ & & \\
\hline IM-2007-32071 & 1 & P. pseudogranulosus & EBISCO & DW2525 & S Nova Bank & $22^{\circ} 48^{\prime} \mathrm{S}, 159^{\circ} 23^{\prime} \mathrm{E} ; 408-410$ & & \\
\hline IM-2007-32072 & 1 & P. pseudogranulosus & EBISCO & DW2528 & S Nova Bank & $22^{\circ} 49^{\prime} \mathrm{S}, 159^{\circ} 23^{\prime} \mathrm{E} ; 320-345$ & & \\
\hline IM-2007-32073 & 1 & P. pseudogranulosus & EBISCO & DW2528 & S Nova Bank & $22^{\circ} 49^{\prime} \mathrm{S}, 159^{\circ} 23^{\prime} \mathrm{E} ; 320-345$ & & \\
\hline IM-2007-32074 & 1 & P. pseudogranulosus & EBISCO & DW2528 & S Nova Bank & $22^{\circ} 49^{\prime} \mathrm{S}, 159^{\circ} 23^{\prime} \mathrm{E} ; 320-345$ & & \\
\hline IM-2007-32075 & 1 & P. pseudogranulosus & EBISCO & DW2528 & S Nova Bank & $22^{\circ} 49^{\prime} \mathrm{S}, 159^{\circ} 23^{\prime} \mathrm{E} ; 320-345$ & & \\
\hline IM-2007-32076 & 1 & P. pseudogranulosus & EBISCO & CP2529 & S Nova Bank & $22^{\circ} 47^{\prime} \mathrm{S}, 159^{\circ} 23^{\prime} \mathrm{E} ; 330-340$ & & \\
\hline IM-2007-32077 & 1 & P. pseudogranulosus & EBISCO & CP2529 & S Nova Bank & $22^{\circ} 47^{\prime} \mathrm{S}, 159^{\circ} 23^{\prime} \mathrm{E} ; 330-340$ & & \\
\hline IM-2007-32078 & 1 & P. pseudogranulosus & EBISCO & CP2529 & S Nova Bank & $22^{\circ} 47^{\prime} \mathrm{S}, 159^{\circ} 23^{\prime} \mathrm{E} ; 330-340$ & & \\
\hline IM-2007-32079 & 1 & P. pseudogranulosus & EBISCO & CP2529 & S Nova Bank & $22^{\circ} 47^{\prime} \mathrm{S}, 159^{\circ} 23^{\prime} \mathrm{E} ; 330-340$ & & \\
\hline IM-2007-32080 & 1 & P. pseudogranulosus & EBISCO & CP2529 & S Nova Bank & $22^{\circ} 47^{\prime} \mathrm{S}, 159^{\circ} 23^{\prime} \mathrm{E} ; 330-340$ & & \\
\hline IM-2007-32081 & 1 & P. pseudogranulosus & EBISCO & CP2529 & S Nova Bank & $22^{\circ} 47^{\prime} \mathrm{S}, 159^{\circ} 23^{\prime} \mathrm{E} ; 330-340$ & & \\
\hline IM-2007-32082 & 1 & P. pseudogranulosus & EBISCO & CP2529 & S Nova Bank & $22^{\circ} 47^{\prime} \mathrm{S}, 159^{\circ} 23^{\prime} \mathrm{E} ; 330-340$ & & \\
\hline IM-2007-32083 & 1 & P. deforgesi & EBISCO & CP2593 & Chesterfield & $19^{\circ} 43^{\prime} \mathrm{S}, 158^{\circ} 32^{\prime} \mathrm{E} ; 300-323$ & PLERO004-15 & KR087207 \\
\hline IM-2007-32084 & 1 & P. deforgesi & EBISCO & СР2593 & Chesterfield & $19^{\circ} 43^{\prime} \mathrm{S}, 158^{\circ} 32^{\prime} \mathrm{E} ; 300-323$ & & \\
\hline IM-2007-32085 & 1 & P. deforgesi & EBISCO & CP2593 & Chesterfield & $19^{\circ} 43^{\prime} \mathrm{S}, 158^{\circ} 32^{\prime} \mathrm{E} ; 300-323$ & PLERO005-15 & KR087215 \\
\hline IM-2007-32086 & 1 & P. deforgesi & EBISCO & CP2593 & Chesterfield & $19^{\circ} 43^{\prime} \mathrm{S}, 158^{\circ} 32^{\prime} \mathrm{E} ; 300-323$ & & \\
\hline IM-2007-32087 & 1 & P. deforgesi & EBISCO & CP2593 & Chesterfield & $19^{\circ} 43^{\prime} \mathrm{S}, 158^{\circ} 32^{\prime} \mathrm{E} ; 300-323$ & PLERO006-15 & KR087214 \\
\hline IM-2007-32088 & 1 & P. deforgesi & EBISCO & CP2593 & Chesterfield & $19^{\circ} 43^{\prime} \mathrm{S}, 158^{\circ} 32^{\prime} \mathrm{E} ; 300-323$ & PLERO007-15 & KR087213 \\
\hline IM-2007-32089 & 1 & P. deforgesi & EBISCO & CP2593 & Chesterfield & $19^{\circ} 43^{\prime} \mathrm{S}, 158^{\circ} 32^{\prime} \mathrm{E} ; 300-323$ & & \\
\hline IM-2007-32090 & 1 & P. deforgesi & EBISCO & CР2593 & Chesterfield & $19^{\circ} 43^{\prime} \mathrm{S}, 158^{\circ} 32^{\prime} \mathrm{E} ; 300-323$ & PLERO008-15 & KR087212 \\
\hline IM-2007-32091 & 1 & P. deforgesi & EBISCO & CP2593 & Chesterfield & $19^{\circ} 43^{\prime} \mathrm{S}, 158^{\circ} 32^{\prime} \mathrm{E} ; 300-323$ & PLERO009-15 & KR087211 \\
\hline
\end{tabular}


ANSEEUW P. et al., New species of Pleurotomariidae

\begin{tabular}{|c|c|c|c|c|c|c|c|c|}
\hline MNHN ID & $\mathbf{N}$ & SPECIES & MNHN EXPEDITION & STATION & GENERAL AREA & COORDINATES; DEPTH (m) & BOLD ID & GENBANK \# \\
\hline IM-2007-34677 & 1 & P. wareni & CONCALIS & DW2946 & Grand Passage & $19^{\circ} 02^{\prime} \mathrm{S}, 163^{\circ} 27^{\prime} \mathrm{E} ; 276-277$ & PLERO024-15 & KR087219 \\
\hline IM-2007-34678 & 1 & P. wareni & CONCALIS & DW2946 & Grand Passage & $19^{\circ} 02^{\prime} \mathrm{S}, 163^{\circ} 27^{\prime} \mathrm{E} ; 276-277$ & & \\
\hline IM-2007-34679 & 1 & P. wareni & CONCALIS & DW3025 & Grand Passage & $18^{\circ} 57^{\prime} \mathrm{S}, 163^{\circ} 23^{\prime} \mathrm{E} ; 396-400$ & & \\
\hline IM-2007-34680 & 1 & P. wareni & CONCALIS & DW2944 & Grand Passage & $18^{\circ} 59^{\prime} \mathrm{S}, 163^{\circ} 24^{\prime} \mathrm{E} ; 320-335$ & PLERO025-15 & KR087228 \\
\hline IM-2007-34681 & 1 & P. wareni & CONCALIS & DW2949 & Grand Passage & $19^{\circ} 00^{\prime} \mathrm{S}, 163^{\circ} 28^{\prime} \mathrm{E} ; 261-272$ & & \\
\hline IM-2007-34682 & 1 & P. wareni & CONCALIS & DW3024 & Grand Passage & $18^{\circ} 57^{\prime} \mathrm{S}, 163^{\circ} 22^{\prime} \mathrm{E} ; 349-370$ & PLERO018-15 & KR087224 \\
\hline IM-2007-34683 & 1 & P. wareni & CONCALIS & DW2941 & Grand Passage & $19^{\circ} 3^{\prime} \mathrm{S}, 163^{\circ} 27^{\prime} \mathrm{E} ; 271-272$ & & \\
\hline IM-2007-34684 & 1 & P. wareni & CONCALIS & DW2973 & Grand Passage & $18^{\circ} 14^{\prime} \mathrm{S}, 163^{\circ} 06^{\prime} \mathrm{E} ; 275-288$ & PLERO019-15 & KR087223 \\
\hline IM-2007-34685 & 1 & P. wareni & CONCALIS & DW2943 & Grand Passage & $18^{\circ} 57^{\prime} \mathrm{S}, 163^{\circ} 23^{\prime} \mathrm{E} ; 380-430$ & PLERO020-15 & KR087222 \\
\hline IM-2007-34686 & 1 & P. wareni & CONCALIS & DW2973 & Grand Passage & $18^{\circ} 14^{\prime} \mathrm{S}, 163^{\circ} 06^{\prime} \mathrm{E} ; 275-288$ & PLERO021-15 & KR087221 \\
\hline IM-2007-34687 & 1 & P. wareni & CONCALIS & DW2974 & Grand Passage & $18^{\circ} 15^{\prime} \mathrm{S}, 163^{\circ} 06^{\prime} \mathrm{E} ; 283-326$ & & \\
\hline IM-2007-34688 & 1 & P. wareni & CONCALIS & CР2975 & Grand Passage & $18^{\circ} 15^{\prime} \mathrm{S}, 163^{\circ} 06^{\prime} \mathrm{E} ; 297-316$ & PLERO022-15 & KR087220 \\
\hline IM-2007-34689 & 1 & P. wareni & CONCALIS & DW2977 & Grand Passage & $18^{\circ} 15^{\prime} \mathrm{S}, 163^{\circ} 07^{\prime} \mathrm{E} ; 326-368$ & & \\
\hline IM-2007-34690 & 1 & P. wareni & CONCALIS & DW2977 & Grand Passage & $18^{\circ} 15^{\prime} \mathrm{S}, 163^{\circ} 07^{\prime} \mathrm{E} ; 326-368$ & PLERO023-15 & KR087229 \\
\hline IM-2007-34691 & 1 & P. wareni & CONCALIS & DW2977 & Grand Passage & $18^{\circ} 15^{\prime} \mathrm{S}, 163^{\circ} 07^{\prime} \mathrm{E} ; 326-368$ & & \\
\hline IM-2007-36300 & 1 & P. caledonicus & TERRASSES & DW3101 & Norfolk Ridge & $22^{\circ} 59^{\prime} \mathrm{S}, 168^{\circ} 21^{\prime} \mathrm{E} ; 320-390$ & PLERO044-15 & KR087186 \\
\hline IM-2007-36301 & 1 & P. caledonicus & TERRASSES & DW3075 & Norfolk Ridge & $23^{\circ} 17^{\prime} \mathrm{S}, 168^{\circ} 14^{\prime} \mathrm{E} ; 270$ & PLERO040-15 & KR087190 \\
\hline IM-2007-36302 & 1 & P. caledonicus & TERRASSES & DW3075 & Norfolk Ridge & $23^{\circ} 17^{\prime} \mathrm{S}, 168^{\circ} 14^{\prime} \mathrm{E} ; 270$ & PLERO041-15 & KR087189 \\
\hline IM-2007-36303 & 1 & P. caledonicus & TERRASSES & DW3035 & Loyalty Ridge & $22^{\circ} 41^{\prime} \mathrm{S}, 168^{\circ} 56^{\prime} \mathrm{E} ; 790-800$ & PLERO038-15 & KR087192 \\
\hline IM-2007-36304 & 1 & P. caledonicus & TERRASSES & DW3035 & Loyalty Ridge & $22^{\circ} 41^{\prime} \mathrm{S}, 168^{\circ} 56^{\prime} \mathrm{E} ; 790-800$ & & \\
\hline IM-2007-36455 & 1 & P. caledonicus & TERRASSES & DW3075 & Norfolk Ridge & $23^{\circ} 17^{\prime} \mathrm{S}, 168^{\circ} 14^{\prime} \mathrm{E} ; 270$ & PLERO042-15 & KR087188 \\
\hline IM-2007-36456 & 1 & P. wareni & TERRASSES & DW3101 & Norfolk Ridge & $22^{\circ} 59^{\prime} \mathrm{S}, 168^{\circ} 21^{\prime} \mathrm{E} ; 320-390$ & PLERO045-15 & KR087227 \\
\hline IM-2007-36457 & 1 & P. caledonicus & TERRASSES & DW3075 & Norfolk Ridge & $23^{\circ} 17^{\prime} \mathrm{S}, 168^{\circ} 14^{\prime} \mathrm{E} ; 270$ & & \\
\hline IM-2007-36458 & 1 & P. caledonicus & TERRASSES & DW3073 & Norfolk Ridge & $23^{\circ} 17^{\prime} \mathrm{S}, 168^{\circ} 14^{\prime} \mathrm{E} ; 250-270$ & PLERO039-15 & KR087191 \\
\hline IM-2007-36459 & 1 & P. caledonicus & TERRASSES & DW3076 & Norfolk Ridge & $23^{\circ} 14^{\prime} \mathrm{S}, 168^{\circ} 13^{\prime} \mathrm{E} ; 390-570$ & PLERO043-15 & KR087187 \\
\hline IM-2007-36460 & 1 & P. wareni & TERRASSES & DW3101 & Norfolk Ridge & $22^{\circ} 59^{\prime} \mathrm{S}, 168^{\circ} 21^{\prime} \mathrm{E} ; 320-390$ & PLERO046-15 & KR087226 \\
\hline IM-2007-36461 & 1 & P. wareni & TERRASSES & DW3101 & Norfolk Ridge & $22^{\circ} 59^{\prime} \mathrm{S}, 168^{\circ} 21^{\prime} \mathrm{E} ; 320-390$ & PLERO047-15 & KR087225 \\
\hline IM-2007-36462 & 1 & P. wareni & TERRASSES & DW3101 & Norfolk Ridge & $22^{\circ} 59^{\prime} \mathrm{S}, 168^{\circ} 21^{\prime} \mathrm{E} ; 320-390$ & & \\
\hline IM-2007-36463 & 1 & P. caledonicus & TERRASSES & DW3110 & Norfolk Ridge & $23^{\circ} 02^{\prime} \mathrm{S}, 168^{\circ} 16^{\prime} \mathrm{E} ; 270-310$ & PLERO048-15 & KR087182 \\
\hline IM-2007-36505 & 1 & P. caledonicus & TERRASSES & DW3127 & Ile des Pins & $23^{\circ} 00^{\prime} \mathrm{S}, 167^{\circ} 16^{\prime} \mathrm{E} ; 400-420$ & PLERO052-15 & KR087178 \\
\hline IM-2007-36519 & 1 & P. caledonicus & TERRASSES & DW3127 & Ile des Pins & $23^{\circ} 00^{\prime} \mathrm{S}, 167^{\circ} 16^{\prime} \mathrm{E} ; 400-420$ & PLERO053-15 & KR087177 \\
\hline IM-2007-36525 & 1 & P. caledonicus & TERRASSES & DW3123 & Ile des Pins & $22^{\circ} 53^{\prime} \mathrm{S}, 167^{\circ} 13^{\prime} \mathrm{E} ; 420-450$ & & \\
\hline IM-2007-36541 & 1 & P. caledonicus & TERRASSES & DW3122 & Ile des Pins & $22^{\circ} 47^{\prime} \mathrm{S}, 167^{\circ} 12^{\prime} \mathrm{E} ; 390-410$ & PLERO034-15 & KR087185 \\
\hline IM-2007-36542 & 1 & P. caledonicus & TERRASSES & DW3122 & Ile des Pins & $22^{\circ} 47^{\prime} \mathrm{S}, 167^{\circ} 12^{\prime} \mathrm{E} ; 390-410$ & PLERO049-15 & KR087181 \\
\hline IM-2007-36545 & 1 & P. caledonicus & TERRASSES & DW3124 & Ile des Pins & $22^{\circ} 54^{\prime} \mathrm{S}, 167^{\circ} 15^{\prime} \mathrm{E} ; 460$ & & \\
\hline IM-2007-36546 & 1 & P. caledonicus & TERRASSES & DW3123 & Ile des Pins & $22^{\circ} 53^{\prime} \mathrm{S}, 167^{\circ} 13^{\prime} \mathrm{E} ; 420-450$ & PLERO051-15 & KR087179 \\
\hline IM-2007-36557 & 1 & P. caledonicus & TERRASSES & DW3122 & Ile des Pins & $22^{\circ} 47^{\prime} \mathrm{S}, 167^{\circ} 12^{\prime} \mathrm{E} ; 390-410$ & PLERO050-15 & KR087180 \\
\hline IM-2007-36577 & 1 & P. caledonicus & TERRASSES & DW3127 & Ile des Pins & $23^{\circ} 00^{\prime} \mathrm{S}, 167^{\circ} 16^{\prime} \mathrm{E} ; 400-420$ & & \\
\hline IM-2007-36592 & 1 & P. caledonicus & TERRASSES & СР 3048 & Norfolk Ridge & $23^{\circ} 44^{\prime} \mathrm{S}, 168^{\circ} 16^{\prime} \mathrm{E} ; 380-400$ & PLERO035-15 & KR087173 \\
\hline IM-2007-36595 & 1 & P. caledonicus & TERRASSES & DW3127 & Ile des Pins & $23^{\circ} 00^{\prime} \mathrm{S}, 167^{\circ} 16^{\prime} \mathrm{E} ; 400-420$ & PLERO054-15 & KR087176 \\
\hline IM-2007-36596 & 1 & P. caledonicus & TERRASSES & CP3052 & Norfolk Ridge & $23^{\circ} 49^{\prime} \mathrm{S}, 168^{\circ} 16^{\prime} \mathrm{E} ; 500-800$ & PLERO037-15 & KR087171 \\
\hline IM-2007-36822 & 1 & P. caledonicus & TERRASSES & СР 3049 & Norfolk Ridge & $23^{\circ} 42^{\prime} \mathrm{S}, 168^{\circ} 16^{\prime} \mathrm{E} ; 380-410$ & PLERO036-15 & KR087172 \\
\hline IM-2009-7476 & 1 & P. caledonicus & NORFOLK 2 & DW2156 & Ile des Pins & $22^{\circ} 54^{\prime} \mathrm{S}, 167^{\circ} 15^{\prime} \mathrm{E} ; 468-500$ & PLERO003-15 & KR087183 \\
\hline IM-2009-7477 & 1 & P. caledonicus & NORFOLK 2 & DW2156 & Ile des Pins & $22^{\circ} 54^{\prime} \mathrm{S}, 167^{\circ} 15^{\prime} \mathrm{E} ; 468-500$ & & \\
\hline IM-2009-7478 & 1 & P. caledonicus & NORFOLK 2 & DW2156 & Ile des Pins & $22^{\circ} 54^{\prime} \mathrm{S}, 167^{\circ} 15^{\prime} \mathrm{E} ; 468-500$ & PLERO001-15 & KR087174 \\
\hline
\end{tabular}


European Journal of Taxonomy 134: 1-23 (2015)

\begin{tabular}{|c|c|c|c|c|c|c|c|c|}
\hline MNHN ID & $\mathbf{N}$ & SPECIES & MNHN EXPEDITION & STATION & GENERAL AREA & COORDINATES; DEPTH (m) & BOLD ID & GENBANK \# \\
\hline IM-2009-7479 & 1 & P. caledonicus & NORFOLK 2 & DW2156 & Ile des Pins & $22^{\circ} 54^{\prime} \mathrm{S}, 167^{\circ} 15^{\prime} \mathrm{E} ; 468-500$ & & \\
\hline IM-2009-7480 & 1 & P. caledonicus & NORFOLK 2 & DW2156 & Ile des Pins & $22^{\circ} 54^{\prime} \mathrm{S}, 167^{\circ} 15^{\prime} \mathrm{E} ; 468-500$ & PLERO059-15 & KR087184 \\
\hline IM-2009-7481 & 1 & P. caledonicus & NORFOLK 2 & DW2156 & Ile des Pins & $22^{\circ} 54^{\prime} \mathrm{S}, 167^{\circ} 15^{\prime} \mathrm{E} ; 468-500$ & & \\
\hline IM-2009-7482 & 1 & P. caledonicus & NORFOLK 2 & DW2156 & Ile des Pins & $22^{\circ} 54^{\prime} \mathrm{S}, 167^{\circ} 15^{\prime} \mathrm{E} ; 468-500$ & & \\
\hline IM-2009-7483 & 1 & P. caledonicus & NORFOLK 2 & DW2156 & Ile des Pins & $22^{\circ} 54^{\prime} \mathrm{S}, 167^{\circ} 15^{\prime} \mathrm{E} ; 468-500$ & PLERO055-15 & KR087175 \\
\hline IM-2009-7485 & 1 & P. pseudogranulosus & EBISCO & DW2528 & S Nova Bank & $22^{\circ} 49^{\prime} \mathrm{S}, 159^{\circ} 23^{\prime} \mathrm{E} ; 320-345$ & PLERO026-15 & KR087202 \\
\hline IM-2009-7486 & 2 & P. deforgesi & EBISCO & $\mathrm{CP} 2593$ & Chesterfield & $19^{\circ} 43^{\prime} \mathrm{S}, 158^{\circ} 32^{\prime} \mathrm{E} ; 300-323$ & PLERO056-15 & KR087210 \\
\hline IM-2009-7487 & 1 & P. pseudogranulosus & EBISCO & CP2529 & S Nova Bank & $22^{\circ} 47^{\prime} \mathrm{S}, 159^{\circ} 23^{\prime} \mathrm{E} ; 330-340$ & PLERO027-15 & KR087201 \\
\hline IM-2009-7488 & 1 & P. pseudogranulosus & EBISCO & CР2529 & S Nova Bank & $22^{\circ} 47^{\prime} \mathrm{S}, 159^{\circ} 23^{\prime} \mathrm{E} ; 330-340$ & PLERO028-15 & KR087200 \\
\hline IM-2009-7489 & 1 & P. pseudogranulosus & EBISCO & DW2526 & S Nova Bank & $22^{\circ} 46^{\prime} \mathrm{S}, 159^{\circ} 23^{\prime} \mathrm{E} ; 330-340$ & PLERO029-15 & KR087199 \\
\hline IM-2009-7490 & 1 & P. deforgesi & EBISCO & CР2567 & NW Bellona & $20^{\circ} 20^{\prime} \mathrm{S}, 158^{\circ} 42^{\prime} \mathrm{E} ; 395-400$ & & \\
\hline IM-2009-7491 & 1 & P. pseudogranulosus & EBISCO & DW2525 & S Nova Bank & $22^{\circ} 48^{\prime} \mathrm{S}, 159^{\circ} 23^{\prime} \mathrm{E} ; 408-410$ & PLERO030-15 & KR087198 \\
\hline IM-2009-7492 & 1 & P. pseudogranulosus & EBISCO & DW2526 & S Nova Bank & $22^{\circ} 46^{\prime} \mathrm{S}, 159^{\circ} 23^{\prime} \mathrm{E} ; 330-340$ & PLERO031-15 & KR087197 \\
\hline IM-2009-7493 & 1 & P. pseudogranulosus & EBISCO & DW2530 & S Nova Bank & $22^{\circ} 48^{\prime} \mathrm{S}, 159^{\circ} 23^{\prime} \mathrm{E} ; 338-343$ & PLERO057-15 & KR087195 \\
\hline IM-2009-7494 & 1 & P. deforgesi & EBISCO & DW2608 & Chesterfield & $1^{\circ} 33^{\prime} \mathrm{S}, 158^{\circ} 40^{\prime} \mathrm{E}$; 393-396 & PLERO032-15 & KR087209 \\
\hline IM-2009-7495 & 1 & P. pseudogranulosus & EBISCO & СР2494 & Capel Bank & $24^{\circ} 45^{\prime} \mathrm{S}, 159^{\circ} 42^{\prime} \mathrm{E} ; 348-354$ & PLERO033-15 & KR087196 \\
\hline IM-2009-7496 & 1 & P. pseudogranulosus & EBISCO & DW2501 & Capel Bank & $24^{\circ} 50^{\prime} \mathrm{S}, 159^{\circ} 51^{\prime} \mathrm{E} ; 325-520$ & & \\
\hline IM-2009-7497 & 1 & P. caledonicus & NORFOLK 2 & DW2031 & Stylaster Bank & $23^{\circ} 39^{\prime} \mathrm{S}, 167^{\circ} 44^{\prime} \mathrm{E} ; 440$ & & \\
\hline IM-2010-11117 & 7 & P. caledonicus & SMIB 8 & DW197-199 & Ile des Pins & $\begin{array}{c}22^{\circ} 51^{\prime}--22^{\circ} 52^{\prime} \mathrm{S}, \\
168^{\circ} 12^{\prime}-168^{\circ} 13^{\prime} \mathrm{E} ; 408-436\end{array}$ & & \\
\hline IM-2010-11118 & 1 & P. caledonicus & SMIB 8 & DW185 & Antigonia Bank & $23^{\circ} 15^{\prime} \mathrm{S}, 168^{\circ} 04^{\prime} \mathrm{E} ; 311-355$ & & \\
\hline IM-2010-11119 & 27 & P. caledonicus & LITHIST & $\mathrm{CP} 16$ & Jumeau Ouest Bank & $23^{\circ} 43^{\prime} \mathrm{S}, 168^{\circ} 16^{\prime} \mathrm{E} ; 379-391$ & & \\
\hline IM-2010-11120 & 1 & P. caledonicus & LITHIST & DW13 & Jumeau Est Bank & $23^{\circ} 45^{\prime} \mathrm{S}, 168^{\circ} 17^{\prime} \mathrm{E} ; 400$ & & \\
\hline IM-2010-11121 & 1 & P. pseudogranulosus & EBISCO & DW2528 & S Nova Bank & $22^{\circ} 49^{\prime} \mathrm{S}, 159^{\circ} 23^{\prime} \mathrm{E} ; 320-345$ & & \\
\hline IM-2010-11122 & 1 & P. caledonicus & SMIB 2 & DW05 & S Ile des Pins & $22^{\circ} 56^{\prime} \mathrm{S}, 167^{\circ} 15^{\prime} \mathrm{E} ; 398-410$ & & \\
\hline IM-2010-11123 & 1 & P. caledonicus & BIOCAL & DW38 & S Ile des Pins & $23^{\circ} 00^{\prime} \mathrm{S}, 167^{\circ} 15^{\prime} \mathrm{E} ; 360$ & & \\
\hline IM-2010-11124 & 2 & P. caledonicus & BATHUS 3 & DW829 & Norfolk Ridge & $23^{\circ} 21^{\prime} \mathrm{S}, 168^{\circ} 02^{\prime} \mathrm{E} ; 386-390$ & & \\
\hline IM-2010-11125 & 13 & P. caledonicus & BATHUS 3 & CP811 & Norfolk Ridge & $23^{\circ} 41^{\prime} \mathrm{S}, 168^{\circ} 16^{\prime} \mathrm{E} ; 383-408$ & & \\
\hline IM-2010-11126 & 9 & P. caledonicus & BATHUS 3 & DW817 & Norfolk Ridge & $23^{\circ} 42^{\prime} \mathrm{S}, 168^{\circ} 16^{\prime} \mathrm{E} ; 405-410$ & & \\
\hline IM-2010-11127 & 3 & P. caledonicus & BATHUS 3 & DW818 & Norfolk Ridge & $23^{\circ} 44^{\prime} \mathrm{S}, 168^{\circ} 16^{\prime} \mathrm{E} ; 394-401$ & & \\
\hline IM-2010-11128 & 1 & P. caledonicus & BATHUS 3 & DW829 & Norfolk Ridge & $23^{\circ} 21^{\prime} \mathrm{S}, 168^{\circ} 02^{\prime} \mathrm{E} ; 386-390$ & & \\
\hline IM-2010-11129 & 5 & P. caledonicus & NORFOLK 2 & СР2048 & Jumeau Est Bank & $23^{\circ} 44^{\prime} \mathrm{S}, 168^{\circ} 16^{\prime} \mathrm{E} ; 380-389$ & & \\
\hline IM-2010-11130 & 4 & P. caledonicus & NORFOLK 2 & DW2049 & Jumeau Est Bank & $23^{\circ} 43^{\prime} \mathrm{S}, 168^{\circ} 15^{\prime} \mathrm{E} ; 470-621$ & & \\
\hline IM-2010-11131 & 7 & P. caledonicus & NORFOLK 2 & CP2050 & Jumeau Est Bank & $23^{\circ} 42^{\prime} \mathrm{S}, 168^{\circ} 16^{\prime} \mathrm{E} ; 377$ & & \\
\hline IM-2010-11132 & 2 & P. caledonicus & NORFOLK 2 & DW2052 & Jumeau Est Bank & $23^{\circ} 42^{\prime} \mathrm{S}, 168^{\circ} 15^{\prime} \mathrm{E} ; 473-525$ & & \\
\hline IM-2010-11133 & 1 & P. caledonicus & NORFOLK 2 & DW2108 & Jumeau Est Bank & $23^{\circ} 47^{\prime} \mathrm{S}, 168^{\circ} 17^{\prime} \mathrm{E} ; 403-440$ & & \\
\hline IM-2010-11134 & 1 & P. caledonicus & NORFOLK 2 & $\mathrm{CP} 2114$ & Jumeau Est Bank & $23^{\circ} 45^{\prime} \mathrm{S}, 168^{\circ} 17^{\prime} \mathrm{E}$; 390-398 & & \\
\hline IM-2010-11135 & 8 & P. caledonicus & NORFOLK 2 & $\mathrm{CH} 2115$ & Jumeau Est Bank & $23^{\circ} 45^{\prime} \mathrm{S}, 168^{\circ} 17^{\prime} \mathrm{E} ; 377-401$ & & \\
\hline IM-2010-11136 & 2 & P. caledonicus & NORFOLK 2 & CP2118 & Antigonia Bank & $23^{\circ} 23^{\prime} \mathrm{S}, 168^{\circ} 01^{\prime} \mathrm{E} ; 383-393$ & & \\
\hline IM-2010-11137 & 3 & P. caledonicus & NORFOLK 2 & DW2126 & Crypthelia Bank & $23^{\circ} 16^{\prime} \mathrm{S}, 168^{\circ} 14^{\prime} \mathrm{E} ; 398-550$ & & \\
\hline IM-2010-11138 & 6 & P. caledonicus & NORFOLK 2 & DW2155 & Ile des Pins & $22^{\circ} 52^{\prime} \mathrm{S}, 167^{\circ} 13^{\prime} \mathrm{E} ; 453-455$ & & \\
\hline IM-2010-11139 & 4 & P. caledonicus & NORFOLK 2 & СР2153 & Ile des Pins & $22^{\circ} 47^{\prime} \mathrm{S}, 167^{\circ} 12^{\prime} \mathrm{E} ; 395-400$ & & \\
\hline IM-2010-11140 & 4 & P. caledonicus & NORFOLK 2 & DW2156 & Ile des Pins & $22^{\circ} 54^{\prime} \mathrm{S}, 167^{\circ} 15^{\prime} \mathrm{E} ; 468-500$ & & \\
\hline IM-2010-11141 & 1 & P. caledonicus & NORFOLK 2 & DW2023 & Brachiopode Bank & $23^{\circ} 27^{\prime} \mathrm{S}, 167^{\circ} 51^{\prime} \mathrm{E} ; 282-297$ & & \\
\hline IM-2010-11142 & 1 & P. caledonicus & NORFOLK 2 & DW2027 & Brachiopode Bank & $23^{\circ} 26^{\prime} \mathrm{S}, 167^{\circ} 51^{\prime} \mathrm{E} ; 465-650$ & & \\
\hline IM-2010-11143 & 2 & P. wareni & BATHUS 4 & DW926 & Grand Passage & $18^{\circ} 57^{\prime} \mathrm{S}, 163^{\circ} 25^{\prime} \mathrm{E} ; 325-330$ & & \\
\hline
\end{tabular}


ANSEEUW P. et al., New species of Pleurotomariidae

\begin{tabular}{|c|c|c|c|c|c|c|c|c|}
\hline MNHN ID & $\mathbf{N}$ & SPECIES & MNHN EXPEDITION & STATION & GENERAL AREA & COORDINATES; DEPTH (m) & BOLD ID & GENBANK \# \\
\hline IM-2010-11144 & 1 & P. wareni & BATHUS 4 & DW925 & Grand Passage & $18^{\circ} 55^{\prime} \mathrm{S}, 163^{\circ} 24^{\prime} \mathrm{E} ; 370-405$ & & \\
\hline IM-2010-11145 & 2 & P. caledonicus & SMIB 8 & DW185 & Antigonia Bank & $23^{\circ} 15^{\prime} \mathrm{S}, 168^{\circ} 04^{\prime} \mathrm{E} ; 311-355$ & & \\
\hline IM-2010-11146 & 2 & P. caledonicus & SMIB 8 & DW179 & Jumeau Est Bank & $23^{\circ} 46^{\prime} \mathrm{S}, 168^{\circ} 17^{\prime} \mathrm{E} ; 400-405$ & & \\
\hline IM-2010-11147 & 14 & P. caledonicus & SMIB 8 & DW199 & Ile des Pins & $22^{\circ} 52^{\prime} \mathrm{S}, 167^{\circ} 12^{\prime} \mathrm{E} ; 408-410$ & & \\
\hline IM-2010-11148 & 8 & P. caledonicus & SMIB 8 & DW197 & Ile des Pins & $22^{\circ} 52^{\prime} \mathrm{S}, 167^{\circ} 13^{\prime} \mathrm{E} ; 414-436$ & & \\
\hline IM-2010-11149 & 2 & P. caledonicus & SMIB 8 & DW198 & Ile des Pins & $22^{\circ} 52^{\prime} \mathrm{S}, 167^{\circ} 13^{\prime} \mathrm{E} ; 414-430$ & & \\
\hline IM-2010-11150 & 8 & P. caledonicus & NORFOLK 1 & DW1704 & Norfolk Ridge & $23^{\circ} 47^{\prime} \mathrm{S}, 168^{\circ} 17^{\prime} \mathrm{E} ; 400-420$ & & \\
\hline IM-2010-11151 & 10 & P. caledonicus & NORFOLK 1 & DW1657 & Norfolk Ridge & $23^{\circ} 26^{\prime} \mathrm{S}, 167^{\circ} 50^{\prime} \mathrm{E} ; 305-332$ & & \\
\hline IM-2010-11152 & 36 & P. caledonicus & NORFOLK 1 & DW1658 & Norfolk Ridge & $23^{\circ} 27^{\prime} \mathrm{S}, 167^{\circ} 50^{\prime} \mathrm{E} ; 320-336$ & & \\
\hline IM-2010-11153 & 8 & P. caledonicus & NORFOLK 1 & CP1705 & Norfolk Ridge & $23^{\circ} 45^{\prime} \mathrm{S}, 168^{\circ} 16^{\prime} \mathrm{E} ; 400-463$ & & \\
\hline IM-2010-11154 & 13 & P. caledonicus & NORFOLK 1 & DW1733 & Norfolk Ridge & $22^{\circ} 57^{\prime} \mathrm{S}, 167^{\circ} 16^{\prime} \mathrm{E} ; 427-433$ & & \\
\hline IM-2010-11155 & 5 & P. caledonicus & NORFOLK 1 & CP1708 & Norfolk Ridge & $23^{\circ} 41^{\prime} \mathrm{S}, 168^{\circ} 15^{\prime} \mathrm{E} ; 381-384$ & & \\
\hline IM-2010-11156 & 9 & P. caledonicus & NORFOLK 1 & DW1709 & Norfolk Ridge & $23^{\circ} 43^{\prime} \mathrm{S}, 168^{\circ} 16^{\prime} \mathrm{E} ; 380-389$ & & \\
\hline IM-2010-11157 & 4 & P. caledonicus & NORFOLK 1 & DW1710 & Norfolk Ridge & $23^{\circ} 45^{\prime} \mathrm{S}, 168^{\circ} 17^{\prime} \mathrm{E}$; 386-426 & & \\
\hline IM-2010-11158 & 13 & P. caledonicus & NORFOLK 1 & DW1707 & Norfolk Ridge & $23^{\circ} 41^{\prime} \mathrm{S}, 168^{\circ} 15^{\prime} \mathrm{E}$; $381-493$ & & \\
\hline IM-2010-11159 & 11 & P. caledonicus & NORFOLK 1 & DW1738 & Norfolk Ridge & $22^{\circ} 51^{\prime} \mathrm{S}, 167^{\circ} 12^{\prime} \mathrm{E} ; 340-381$ & & \\
\hline IM-2010-11160 & 6 & P. caledonicus & NORFOLK 1 & DW1734 & Norfolk Ridge & $22^{\circ} 54^{\prime} \mathrm{S}, 167^{\circ} 13^{\prime} \mathrm{E}$; $403-429$ & & \\
\hline IM-2010-11161 & 23 & P. caledonicus & NORFOLK 1 & DW1737 & Norfolk Ridge & $22^{\circ} 51^{\prime} \mathrm{S}, 167^{\circ} 10^{\prime} \mathrm{E} ; 400$ & & \\
\hline IM-2010-11162 & 14 & P. caledonicus & NORFOLK 1 & DW1736 & Norfolk Ridge & $22^{\circ} 52^{\prime} \mathrm{S}, 167^{\circ} 12^{\prime} \mathrm{E} ; 383-407$ & & \\
\hline IM-2010-11163 & 3 & P. caledonicus & NORFOLK 1 & DW1735 & Norfolk Ridge & $22^{\circ} 53^{\prime} \mathrm{S}, 167^{\circ} 13^{\prime} \mathrm{E} ; 415-445$ & & \\
\hline IM-2010-11164 & 5 & P. caledonicus & NORFOLK 1 & DW1739 & Norfolk Ridge & $22^{\circ} 51^{\prime} \mathrm{S}, 167^{\circ} 14^{\prime} \mathrm{E} ; 404-448$ & & \\
\hline IM-2010-11165 & 1 & P. caledonicus & NORFOLK 1 & CP1669 & Norfolk Ridge & $23^{\circ} 41^{\prime} \mathrm{S}, 168^{\circ} 01^{\prime} \mathrm{E} ; 302-325$ & & \\
\hline IM-2010-11166 & 1 & P. caledonicus & NORFOLK 1 & CP1715 & Norfolk Ridge & $23^{\circ} 22^{\prime} \mathrm{S}, 168^{\circ} 03^{\prime} \mathrm{E} ; 270-312$ & & \\
\hline IM-2010-11167 & 1 & P. caledonicus & NORFOLK 1 & DW1653 & Norfolk Ridge & $23^{\circ} 26^{\prime} \mathrm{S}, 1^{6} 67^{\circ} 51^{\prime} \mathrm{E} ; 328-340$ & & \\
\hline IM-2010-11168 & 1 & P. caledonicus & NORFOLK 1 & CP1711 & Norfolk Ridge & $23^{\circ} 48^{\prime} \mathrm{S}, 168^{\circ} 17^{\prime} \mathrm{E}$; 409-439 & & \\
\hline IM-2010-11169 & 1 & P. caledonicus & NORFOLK 1 & DW1712 & Norfolk Ridge & $23^{\circ} 22^{\prime} \mathrm{S}, 168^{\circ} 03^{\prime} \mathrm{E} ; 180-250$ & & \\
\hline IM-2010-11170 & 1 & P. caledonicus & NORFOLK 1 & CP1671 & Norfolk Ridge & $23^{\circ} 42^{\prime} \mathrm{S}, 168^{\circ} 01^{\prime} \mathrm{E} ; 320-397$ & & \\
\hline IM-2010-11171 & 1 & P. caledonicus & NORFOLK 1 & DW1652 & Norfolk Ridge & $23^{\circ} 27^{\prime} \mathrm{S}, 167^{\circ} 51^{\prime} \mathrm{E} ; 290-378$ & & \\
\hline IM-2010-11172 & 1 & P. caledonicus & LITHIST & $\mathrm{CP} 17$ & Jumeau Ouest Bank & $23^{\circ} 41^{\prime} \mathrm{S}, 168^{\circ} 01^{\prime} \mathrm{E} ; 247-281$ & & \\
\hline IM-2010-11173 & 15 & P. caledonicus & LITHIST & $\mathrm{CP} 15$ & Jumeau Ouest Bank & $23^{\circ} 40^{\prime} \mathrm{S}, 168^{\circ} 15^{\prime} \mathrm{E} ; 389-404$ & & \\
\hline IM-2010-11174 & 15 & P. caledonicus & LITHIST & DW13 & Jumeau Est Bank & $23^{\circ} 45^{\prime} \mathrm{S}, 168^{\circ} 17^{\prime} \mathrm{E} ; 400$ & & \\
\hline IM-2010-11175 & 21 & P. caledonicus & LITHIST & CP14 & Jumeau Ouest Bank & $23^{\circ} 42^{\prime} \mathrm{S}, 168^{\circ} 16^{\prime} \mathrm{E} ; 378-402$ & & \\
\hline IM-2010-11176 & 1 & P. wareni & LITHIST & CP16 & Jumeau Ouest Bank & $23^{\circ} 43^{\prime} \mathrm{S}, 168^{\circ} 16^{\prime} \mathrm{E} ; 379-391$ & & \\
\hline IM-2010-11177 & 1 & P. caledonicus & TERRASSES & DW3127 & Ile des Pins & $23^{\circ} 00^{\prime} \mathrm{S}, 167^{\circ} 16^{\prime} \mathrm{E} ; 400-420$ & & \\
\hline IM-2010-11178 & 1 & P. caledonicus & TERRASSES & DW3053 & Norfolk Ridge & $23^{\circ} 45^{\prime} \mathrm{S}, 168^{\circ} 16^{\prime} \mathrm{E}$; $410-440$ & & \\
\hline IM-2010-11179 & 2 & P. caledonicus & TERRASSES & СР3049 & Norfolk Ridge & $23^{\circ} 42^{\prime} \mathrm{S}, 168^{\circ} 16^{\prime} \mathrm{E} ; 380-410$ & & \\
\hline IM-2010-11180 & 2 & P. caledonicus & TERRASSES & DW3122 & Ile des Pins & $22^{\circ} 47^{\prime} \mathrm{S}, 167^{\circ} 12^{\prime} \mathrm{E} ; 390-410$ & & \\
\hline IM-2010-11181 & 2 & P. caledonicus & TERRASSES & DW3123 & Ile des Pins & $22^{\circ} 53^{\prime} \mathrm{S}, 167^{\circ} 13^{\prime} \mathrm{E} ; 420-450$ & & \\
\hline IM-2010-11182 & 4 & P. caledonicus & TERRASSES & DW3124 & Ile des Pins & $22^{\circ} 54^{\prime} \mathrm{S}, 167^{\circ} 15^{\prime} \mathrm{E} ; 460$ & & \\
\hline IM-2010-11183 & 3 & P. caledonicus & TERRASSES & СР3048 & Norfolk Ridge & $23^{\circ} 44^{\prime} \mathrm{S}, 168^{\circ} 16^{\prime} \mathrm{E} ; 380-400$ & & \\
\hline IM-2010-11184 & 2 & P. caledonicus & MUSORSTOM 4 & DW222 & S Grande-Terre & $22^{\circ} 58^{\prime} \mathrm{S}, 167^{\circ} 33^{\prime} \mathrm{E} ; 410-440$ & & \\
\hline IM-2010-11185 & 17 & P. caledonicus & & $\begin{array}{c}\text { ACC. } \\
386350\end{array}$ & & & & \\
\hline IM-2010-11186 & 6 & P. caledonicus & SMIB 2 & DW05 & S Ile des Pins & $22^{\circ} 56^{\prime} \mathrm{S}, 167^{\circ} 15^{\prime} \mathrm{E}$; 398-410 & & \\
\hline IM-2010-11187 & 1 & P. caledonicus & BATHUS 3 & DW817 & Norfolk Ridge & $23^{\circ} 42^{\prime} \mathrm{S}, 168^{\circ} 16^{\prime} \mathrm{E} ; 405-410$ & & \\
\hline IM-2010-11188 & 13 & P. caledonicus & BATHUS 2 & DW729 & SW Passe de Dumbéa & $22^{\circ} 52^{\prime} \mathrm{S}, 167^{\circ} 12^{\prime} \mathrm{E} ; 400$ & & \\
\hline IM-2010-11189 & 7 & P. caledonicus & SMIB 1 & DW02 & S Ile des Pins & $22^{\circ} 52^{\prime} \mathrm{S}, 167^{\circ} 13^{\prime} \mathrm{E} ; 415$ & & \\
\hline
\end{tabular}


European Journal of Taxonomy 134: 1-23 (2015)

\begin{tabular}{|c|c|c|c|c|c|c|c|c|}
\hline MNHN ID & $\mathbf{N}$ & SPECIES & MNHN EXPEDITION & STATION & GENERAL AREA & COORDINATES; DEPTH (m) & BOLD ID & GENBANK \# \\
\hline IM-2010-11190 & 3 & P. caledonicus & SMIB 1 & DW07 & S Ile des Pins & $22^{\circ} 55^{\prime} \mathrm{S}, 167^{\circ} 16^{\prime} \mathrm{E} ; 500$ & & \\
\hline IM-2010-11191 & 6 & P. caledonicus & SMIB 1 & DW09 & S Ile des Pins & $22^{\circ} 55^{\prime} \mathrm{S}, 167^{\circ} 15^{\prime} \mathrm{E} ; 450$ & & \\
\hline IM-2010-11192 & 1 & P. caledonicus & BIOCAL & $\mathrm{CP} 42$ & S Ile des Pins & $22^{\circ} 46^{\prime} \mathrm{S}, 167^{\circ} 14^{\prime} \mathrm{E} ; 380$ & & \\
\hline IM-2010-11193 & 1 & P. caledonicus & LAGON & DW1147 & Belep & $19^{\circ} 08^{\prime} \mathrm{S}, 163^{\circ} 30^{\prime} \mathrm{E} ; 210$ & & \\
\hline IM-2010-11194 & 1 & P. caledonicus & VAUBAN & DR15 & & $22^{\circ} 49^{\prime} \mathrm{S}, 167^{\circ} 12^{\prime} \mathrm{E} ; 390-395$ & & \\
\hline IM-2010-11195 & 24 & P. caledonicus & SMIB 2 & DW03 & S Ile des Pins & $22^{\circ} 54^{\prime} \mathrm{S}, 167^{\circ} 14^{\prime} \mathrm{E} ; 412-428$ & & \\
\hline IM-2010-11196 & 3 & P. caledonicus & SMIB 2 & DW01 & S Ile des Pins & $22^{\circ} 53^{\prime} \mathrm{S}, 167^{\circ} 13^{\prime} \mathrm{E} ; 438-444$ & & \\
\hline IM-2010-11197 & 3 & P. caledonicus & SMIB 2 & DW17 & S Ile des Pins & $22^{\circ} 56^{\prime} \mathrm{S}, 167^{\circ} 16^{\prime} \mathrm{E} ; 428-448$ & & \\
\hline IM-2010-11198 & 6 & P. caledonicus & SMIB 2 & DW14 & S Ile des Pins & $22^{\circ} 53^{\prime} \mathrm{S}, 167^{\circ} 13^{\prime} \mathrm{E} ; 405-444$ & & \\
\hline IM-2010-11199 & 4 & P. caledonicus & SMIB 2 & DW08 & S Ile des Pins & $22^{\circ} 53^{\prime} \mathrm{S}, 167^{\circ} 14^{\prime} \mathrm{E} ; 435-447$ & & \\
\hline IM-2010-11200 & 3 & P. caledonicus & SMIB 2 & DW16 & S Ile des Pins & $22^{\circ} 52^{\prime} \mathrm{S}, 167^{\circ} 12^{\prime} \mathrm{E} ; 390$ & & \\
\hline IM-2010-11201 & 2 & P. caledonicus & SMIB 2 & DW07 & S Ile des Pins & $22^{\circ} 56^{\prime} \mathrm{S}, 167^{\circ} 14^{\prime} \mathrm{E} ; 428$ & & \\
\hline IM-2010-11202 & 2 & P. caledonicus & SMIB 2 & DW04 & S Ile des Pins & $22^{\circ} 53^{\prime} \mathrm{S}, 167^{\circ} 14^{\prime} \mathrm{E} ; 410-417$ & & \\
\hline IM-2010-11203 & 1 & P. caledonicus & SMIB 2 & DW15 & S Ile des Pins & $22^{\circ} 53^{\prime} \mathrm{S}, 167^{\circ} 12^{\prime} \mathrm{E} ; 375-402$ & & \\
\hline IM-2010-11204 & 1 & P. caledonicus & SMIB 2 & DW12 & S Ile des Pins & $22^{\circ} 51^{\prime} \mathrm{S}, 167^{\circ} 13^{\prime} \mathrm{E}$; $445-460$ & & \\
\hline IM-2010-11205 & 1 & P. caledonicus & SMIB 2 & DW09 & S Ile des Pins & $22^{\circ} 55^{\prime} \mathrm{S}, 167^{\circ} 16^{\prime} \mathrm{E} ; 475-500$ & & \\
\hline IM-2010-11206 & 1 & P. caledonicus & SMIB 3 & DW26 & SW Ile des Pins & $22^{\circ} 55^{\prime} \mathrm{S}, 167^{\circ} 16^{\prime} \mathrm{E} ; 450$ & & \\
\hline IM-2010-11207 & 1 & P. caledonicus & SMIB 3 & DW20 & Jumeau Ouest Bank & $23^{\circ} 40^{\prime} \mathrm{S}, 168^{\circ} 00^{\prime} \mathrm{E} ; 280$ & & \\
\hline IM-2010-11208 & 1 & P. caledonicus & SMIB 3 & DW27 & SW Ile des Pins & $22^{\circ} 55^{\prime} \mathrm{S}, 167^{\circ} 18^{\prime} \mathrm{E} ; 457$ & & \\
\hline IM-2010-11209 & 1 & P. caledonicus & SMIB 3 & DW28 & SW Ile des Pins & $22^{\circ} 46^{\prime} \mathrm{S}, 167^{\circ} 11^{\prime} \mathrm{E} ; 394$ & & \\
\hline IM-2010-11210 & 1 & P. caledonicus & SMIB 3 & DW06 & Éponge Bank & $24^{\circ} 55^{\prime} \mathrm{S}, 168^{\circ} 21^{\prime} \mathrm{E} ; 505$ & & \\
\hline IM-2010-11211 & 1 & P. caledonicus & SMIB 3 & DW25 & SW Ile des Pins & $22^{\circ} 56^{\prime} \mathrm{S}, 167^{\circ} 15^{\prime} \mathrm{E} ; 437$ & & \\
\hline IM-2010-11212 & 2 & P. caledonicus & CHALCAL 2 & DW82 & Norfolk Ridge & $23^{\circ} 14^{\prime} \mathrm{S}, 168^{\circ} 04^{\prime} \mathrm{E} ; 304$ & & \\
\hline IM-2010-11213 & 1 & P. caledonicus & MUSORSTOM 4 & $\mathrm{CP} 213$ & S Grande-Terre & $22^{\circ} 51^{\prime} \mathrm{S}, 167^{\circ} 12^{\prime} \mathrm{E} ; 405-430$ & & \\
\hline IM-2010-11214 & 1 & P. caledonicus & MUSORSTOM 4 & DW212 & S Grande-Terre & $22^{\circ} 47^{\prime} \mathrm{S}, 167^{\circ} 10^{\prime} \mathrm{E} ; 375-380$ & & \\
\hline IM-2010-11215 & 1 & P. caledonicus & MUSORSTOM 4 & DW229 & S Grande-Terre & $22^{\circ} 52^{\prime} \mathrm{S}, 167^{\circ} 13^{\prime} \mathrm{E} ; 445-460$ & & \\
\hline IM-2010-11216 & 5 & P. caledonicus & MUSORSTOM 4 & $\mathrm{CP} 214$ & S Grande-Terre & $22^{\circ} 54^{\prime} \mathrm{S}, 167^{\circ} 14^{\prime} \mathrm{E} ; 425-440$ & & \\
\hline IM-2010-11217 & 6 & P. caledonicus & SMIB 4 & DW65 & Ile des Pins & $22^{\circ} 56^{\prime} \mathrm{S}, 167^{\circ} 14^{\prime} \mathrm{E} ; 400-420$ & & \\
\hline IM-2010-11218 & 1 & P. caledonicus & SMIB 4 & DW57 & Antigonia Bank & $23^{\circ} 21^{\prime} \mathrm{S}, 168^{\circ} 04^{\prime} \mathrm{E} ; 210-260$ & & \\
\hline IM-2010-11219 & 1 & P. caledonicus & VAUBAN & $\mathrm{DR} 24$ & & $22^{\circ} 48^{\prime} \mathrm{S}, 167^{\circ} 09^{\prime} \mathrm{E} ; 355-360$ & & \\
\hline IM-2010-11220 & 6 & P. caledonicus & VAUBAN & DR15 & & $22^{\circ} 49^{\prime} \mathrm{S}, 167^{\circ} 12^{\prime} \mathrm{E} ; 390-395$ & & \\
\hline IM-2010-11221 & 2 & P. caledonicus & & 444 & & $18^{\circ} 15^{\prime} \mathrm{S}, 162^{\circ} 59^{\prime} \mathrm{E} ; 300-350$ & & \\
\hline IM-2010-11222 & 1 & P. deforgesi & MUSORSTOM 5 & DC361 & Chesterfield & $19^{\circ} 52^{\prime} \mathrm{S}, 158^{\circ} 38^{\prime} \mathrm{E} ; 400$ & & \\
\hline IM-2010-11223 & 3 & P. deforgesi & CHALCAL 1 & DC32 & Chesterfield & $19^{\circ} 43^{\prime} \mathrm{S}, 158^{\circ} 33^{\prime} \mathrm{E} ; 350$ & & \\
\hline IM-2010-11224 & 1 & P. deforgesi & CHALCAL 1 & СР08 & Chesterfield & $19^{\circ} 44^{\prime} \mathrm{S}, 158^{\circ} 35^{\prime} \mathrm{E} ; 348$ & & \\
\hline IM-2010-11225 & 1 & P. deforgesi & MUSORSTOM 5 & DW338 & Chesterfield & $19^{\circ} 52^{\prime} \mathrm{S}, 158^{\circ} 40^{\prime} \mathrm{E} ; 540-580$ & & \\
\hline IM-2010-11226 & 3 & P. deforgesi & MUSORSTOM 5 & DC379 & Chesterfield & $19^{\circ} 53^{\prime} \mathrm{S}, 158^{\circ} 39^{\prime} \mathrm{E} ; 370-400$ & & \\
\hline IM-2010-11227 & 5 & P. deforgesi & MUSORSTOM 5 & DC361 & Chesterfield & $19^{\circ} 52^{\prime} \mathrm{S}, 158^{\circ} 38^{\prime} \mathrm{E} ; 400$ & & \\
\hline IM-2010-11228 & 1 & P. deforgesi & MUSORSTOM 5 & DC361 & Chesterfield & $19^{\circ} 52^{\prime} \mathrm{S}, 158^{\circ} 38^{\prime} \mathrm{E} ; 400$ & & \\
\hline IM-2010-11229 & 1 & P. deforgesi & MUSORSTOM 5 & DW337 & Chesterfield & $19^{\circ} 54^{\prime} \mathrm{S}, 158^{\circ} 38^{\prime} \mathrm{E} ; 412-430$ & & \\
\hline IM-2010-11230 & 1 & P. deforgesi & MUSORSTOM 5 & DC361 & Chesterfield & $19^{\circ} 52^{\prime} \mathrm{S}, 158^{\circ} 38^{\prime} \mathrm{E} ; 400$ & & \\
\hline IM-2010-11231 & 1 & P. deforgesi & MUSORSTOM 5 & DC378 & Chesterfield & $19^{\circ} 54^{\prime} \mathrm{S}, 158^{\circ} 38^{\prime} \mathrm{E} ; 355$ & & \\
\hline IM-2010-11232 & 1 & P. deforgesi & EBISCO & CР2579 & N Bellona & $20^{\circ} 21^{\prime} \mathrm{S}, 158^{\circ} 40^{\prime} \mathrm{E} ; 440-455$ & & \\
\hline IM-2010-11233 & 2 & P. deforgesi & EBISCO & CP2596 & Chesterfield & $19^{\circ} 43^{\prime} \mathrm{S}, 158^{\circ} 37^{\prime} \mathrm{E} ; 382-386$ & & \\
\hline IM-2010-11234 & 1 & P. deforgesi & EBISCO & DW2606 & Chesterfield & $19^{\circ} 37^{\prime} \mathrm{S}, 158^{\circ} 42^{\prime} \mathrm{E} ; 442-443$ & & \\
\hline IM-2010-11235 & 3 & P. deforgesi & EBISCO & DW2564 & NW Bellona & $20^{\circ} 25^{\prime} \mathrm{S}, 158^{\circ} 41^{\prime} \mathrm{E} ; 333-386$ & & \\
\hline
\end{tabular}


ANSEEUW P. et al., New species of Pleurotomariidae

\begin{tabular}{|c|c|c|c|c|c|c|c|c|}
\hline MNHN ID & $\mathbf{N}$ & SPECIES & MNHN EXPEDITION & STATION & GENERAL AREA & COORDINATES; DEPTH (m) & BOLD ID & GENBANK \# \\
\hline IM-2010-11236 & 9 & P. deforgesi & EBISCO & CP2595 & Chesterfield & $19^{\circ} 44^{\prime} \mathrm{S}, 158^{\circ} 35^{\prime} \mathrm{E} ; 345-377$ & & \\
\hline IM-2010-11237 & 3 & P. deforgesi & EBISCO & DW2577 & N Bellona & $20^{\circ} 20^{\prime} \mathrm{S}, 158^{\circ} 39^{\prime} \mathrm{E}$; 399-602 & & \\
\hline IM-2010-11238 & 1 & P. deforgesi & EBISCO & DW2576 & N Bellona & $20^{\circ} 20^{\prime} \mathrm{S}, 158^{\circ} 43^{\prime} \mathrm{E} ; 390-394$ & & \\
\hline IM-2010-11239 & 17 & P. pseudogranulosus & EBISCO & DW2530 & S Nova Bank & $22^{\circ} 48^{\prime} \mathrm{S}, 159^{\circ} 23^{\prime} \mathrm{E} ; 338-343$ & & \\
\hline IM-2010-11240 & 21 & P. pseudogranulosus & EBISCO & CP2531 & S Nova Bank & $22^{\circ} 47^{\prime} \mathrm{S}, 159^{\circ} 23^{\prime} \mathrm{E} ; 330-340$ & & \\
\hline IM-2010-11241 & 2 & P. pseudogranulosus & EBISCO & CP2503 & Capel Bank & $24^{\circ} 48^{\prime} \mathrm{S}, 159^{\circ} 46^{\prime} \mathrm{E} ; 366-380$ & & \\
\hline IM-2010-11242 & 3 & P. pseudogranulosus & EBISCO & DW2533 & N Nova Bank & $22^{\circ} 18^{\prime} \mathrm{S}, 159^{\circ} 28^{\prime} \mathrm{E} ; 360-370$ & & \\
\hline IM-2010-11243 & 1 & P. pseudogranulosus & MUSORSTOM 5 & DW304 & Nova Bank & $22^{\circ} 10^{\prime} \mathrm{S}, 159^{\circ} 25^{\prime} \mathrm{E} ; 385-420$ & & \\
\hline IM-2010-11244 & 1 & P. pseudogranulosus & MUSORSTOM 5 & DW300 & Nova Bank & $22^{\circ} 48^{\prime} \mathrm{S}, 159^{\circ} 24^{\prime} \mathrm{E} ; 450$ & & \\
\hline IM-2010-11245 & 4 & P. pseudogranulosus & MUSORSTOM 5 & DW301 & Nova Bank & $22^{\circ} 07^{\prime} \mathrm{S}, 159^{\circ} 25^{\prime} \mathrm{E} ; 487-610$ & & \\
\hline IM-2010-11246 & 3 & P. pseudogranulosus & MUSORSTOM 5 & DW299 & Nova Bank & $22^{\circ} 48^{\prime} \mathrm{S}, 159^{\circ} 24^{\prime} \mathrm{E} ; 360-390$ & & \\
\hline IM-2010-11301 & 1 & P. wareni & MUSORSTOM 6 & DW407 & Loyalty Ridge & $20^{\circ} 41^{\prime} \mathrm{S}, 167^{\circ} 07^{\prime} \mathrm{E} ; 360$ & & \\
\hline IM-2010-11302 & 3 & P. wareni & HALICAL 1 & DW01 & Grand Passage & $18^{\circ} 56^{\prime} \mathrm{S}, 163^{\circ} 24^{\prime} \mathrm{E} ; 380-400$ & & \\
\hline IM-2010-11303 & 4 & P. wareni & HALICAL 1 & DW04 & Grand Passage & $18^{\circ} 55^{\prime} \mathrm{S}, 163^{\circ} 24^{\prime} \mathrm{E} ; 350-365$ & & \\
\hline IM-2010-11304 & 3 & P. wareni & HALICAL 1 & DW03 & Grand Passage & $18^{\circ} 53^{\prime} \mathrm{S}, 163^{\circ} 24^{\prime} \mathrm{E} ; 350-380$ & & \\
\hline IM-2010-11305 & 1 & P. wareni & MUSORSTOM 4 & $\mathrm{CC} 173$ & Grand Passage & $19^{\circ} 02^{`} \mathrm{~S}, 163^{\circ} 19^{\prime} \mathrm{E} ; 250-290$ & & \\
\hline IM-2010-11306 & 1 & P. wareni & MUSORSTOM 6 & DC402 & Loyalty Ridge & $20^{\circ} 30^{\prime} \mathrm{S}, 166^{\circ} 49^{\prime} \mathrm{E} ; 520$ & & \\
\hline IM-2010-11307 & 1 & P. wareni & BERYX 11 & CP51 & Norfolk Ridge & $23^{\circ} 45^{\prime} \mathrm{S}, 168^{\circ} 17^{\prime} \mathrm{E} ; 390-400$ & & \\
\hline IM-2010-11308 & 1 & P. wareni & TERRASSES & DW3101 & Norfolk Ridge & $22^{\circ} 59^{\prime} \mathrm{S}, 168^{\circ} 21^{\prime} \mathrm{E} ; 320-390$ & & \\
\hline IM-2010-11309 & 1 & P. wareni & TERRASSES & DW3110 & Norfolk Ridge & $23^{\circ} 02^{\prime} \mathrm{S}, 168^{\circ} 16^{\prime} \mathrm{E} ; 270-310$ & & \\
\hline IM-2010-11310 & 1 & P. wareni & NORFOLK 2 & CP2095 & Kaimon Maru Bank & $24^{\circ} 46^{\prime} \mathrm{S}, 168^{\circ} 10^{\prime} \mathrm{E} ; 283-310$ & & \\
\hline IM-2010-11311 & 2 & P. wareni & SMIB 5 & DW85 & NW Walpole & $22^{\circ} 20^{\prime} \mathrm{S}, 168^{\circ} 42^{\prime} \mathrm{E} ; 240-260$ & & \\
\hline IM-2010-11312 & 5 & P. wareni & LITHIST & CP14 & Jumeau Ouest Bank & $23^{\circ} 42^{\prime} \mathrm{S}, 168^{\circ} 16^{\prime} \mathrm{E} ; 378-402$ & & \\
\hline IM-2010-11313 & 2 & P. wareni & SMIB 8 & DW160 & Kaimon Maru Bank & $24^{\circ} 47^{\prime} \mathrm{S}, 168^{\circ} 08^{\prime} \mathrm{E} ; 280-282$ & & \\
\hline IM-2010-11314 & 2 & P. wareni & CONCALIS & DW2968 & Grand Passage & $18^{\circ} 14^{\prime} \mathrm{S}, 163^{\circ} 02^{\prime} \mathrm{E} ; 247-256$ & & \\
\hline IM-2010-11315 & 1 & P. wareni & CONCALIS & СР3006 & Grand Passage & $18^{\circ} 32^{\prime} \mathrm{S}, 163^{\circ} 08^{\prime} \mathrm{E} ; 400$ & & \\
\hline IM-2010-11316 & 2 & P. wareni & CONCALIS & CP2975 & Grand Passage & $18^{\circ} 15^{\prime} \mathrm{S}, 163^{\circ} 06^{\prime} \mathrm{E} ; 297-316$ & & \\
\hline IM-2010-11317 & 2 & P. wareni & CONCALIS & DW2946 & Grand Passage & $19^{\circ} 02^{\prime} \mathrm{S}, 163^{\circ} 27^{\prime} \mathrm{E} ; 276-277$ & & \\
\hline IM-2010-11318 & 4 & P. wareni & BATHUS 4 & DW924 & Grand Passage & $18^{\circ} 55^{\prime} \mathrm{S}, 163^{\circ} 24^{\prime} \mathrm{E} ; 344-360$ & & \\
\hline IM-2010-11319 & 3 & P. wareni & BATHUS 4 & CP938 & Grand Passage & $19^{\circ} 00^{\prime} \mathrm{S}, 163^{\circ} 26^{\prime} \mathrm{E} ; 280-288$ & & \\
\hline IM-2010-11320 & 5 & P. wareni & BATHUS 4 & DW925 & Grand Passage & $18^{\circ} 55^{\prime} \mathrm{S}, 163^{\circ} 24^{\prime} \mathrm{E} ; 370-405$ & & \\
\hline IM-2010-11321 & 1 & P. wareni & BATHUS 4 & DW926 & Grand Passage & $18^{\circ} 57^{\prime} \mathrm{S}, 163^{\circ} 25^{\prime} \mathrm{E} ; 325-330$ & & \\
\hline IM-2010-11322 & 8 & P. wareni & BATHUS 4 & DW931 & Grand Passage & $18^{\circ} 55^{\prime} \mathrm{S}, 163^{\circ} 24^{\prime} \mathrm{E} ; 360-377$ & & \\
\hline IM-2010-11323 & 1 & P. wareni & NORFOLK 1 & DW1709 & Norfolk Ridge & $23^{\circ} 43^{\prime} \mathrm{S}, 168^{\circ} 16^{\prime} \mathrm{E} ; 380-389$ & & \\
\hline IM-2010-11324 & 1 & P. wareni & NORFOLK 1 & DW1658 & Norfolk Ridge & $23^{\circ} 27^{\prime} \mathrm{S}, 167^{\circ} 50^{\prime} \mathrm{E} ; 320-336$ & & \\
\hline IM-2010-11325 & 1 & P. wareni & NORFOLK 1 & DW1729 & Norfolk Ridge & $23^{\circ} 21^{\prime} \mathrm{S}, 168^{\circ} 16^{\prime} \mathrm{E} ; 340-619$ & & \\
\hline IM-2010-11326 & 1 & P. wareni & SMIB 8 & DW189 & Antigonia Bank & $23^{\circ} 18^{\prime} \mathrm{S}, 168^{\circ} 06^{\prime} \mathrm{E} ; 400-402$ & & \\
\hline IM-2010-11327 & 1 & P. wareni & BATHUS 4 & DW924 & Grand Passage & $18^{\circ} 55^{\prime} \mathrm{S}, 163^{\circ} 24^{\prime} \mathrm{E} ; 344-360$ & & \\
\hline IM-2010-11328 & 4 & P. wareni & MUSORSTOM 4 & CP193 & Grand Passage & $18^{\circ} 56^{\prime} \mathrm{S}, 163^{\circ} 23^{\prime} \mathrm{E} ; 430$ & & \\
\hline IM-2010-11329 & 1 & P. wareni & MUSORSTOM 4 & DW196 & Grand Passage & $18^{\circ} 55^{\prime} \mathrm{S}, 163^{\circ} 24^{\prime} \mathrm{E} ; 460$ & & \\
\hline IM-2010-11330 & 1 & P. wareni & MUSORSTOM 4 & DW222 & S Grande-Terre & $22^{\circ} 58^{\prime} \mathrm{S}, 167^{\circ} 33^{\prime} \mathrm{E} ; 410-440$ & & \\
\hline IM-2010-11331 & 1 & P. wareni & MUSORSTOM 6 & DW407 & Loyalty Ridge & $20^{\circ} 41^{\prime} \mathrm{S}, 167^{\circ} 07^{\prime} \mathrm{E} ; 360$ & & \\
\hline IM-2010-11332 & 1 & P. wareni & MUSORSTOM 6 & DW473 & Loyalty Ridge & $21^{\circ} 09^{\prime} \mathrm{S}, 167^{\circ} 55^{\prime} \mathrm{E} ; 236$ & & \\
\hline IM-2010-11334 & 2 & P. wareni & MUSORSTOM 4 & & & 250 & & \\
\hline IM-2010-11335 & 1 & P. wareni & SMIB 6 & DW121 & Grand Passage & $18^{\circ} 58^{\prime} \mathrm{S}, 163^{\circ} 26^{\prime} \mathrm{E} ; 315$ & & \\
\hline IM-2010-11336 & 1 & P. wareni & BATHUS 3 & CP812 & Norfolk Ridge & $23^{\circ} 43^{\prime} \mathrm{S}, 168^{\circ} 16^{\prime} \mathrm{E} ; 391-440$ & & \\
\hline
\end{tabular}


European Journal of Taxonomy 134: 1-23 (2015)

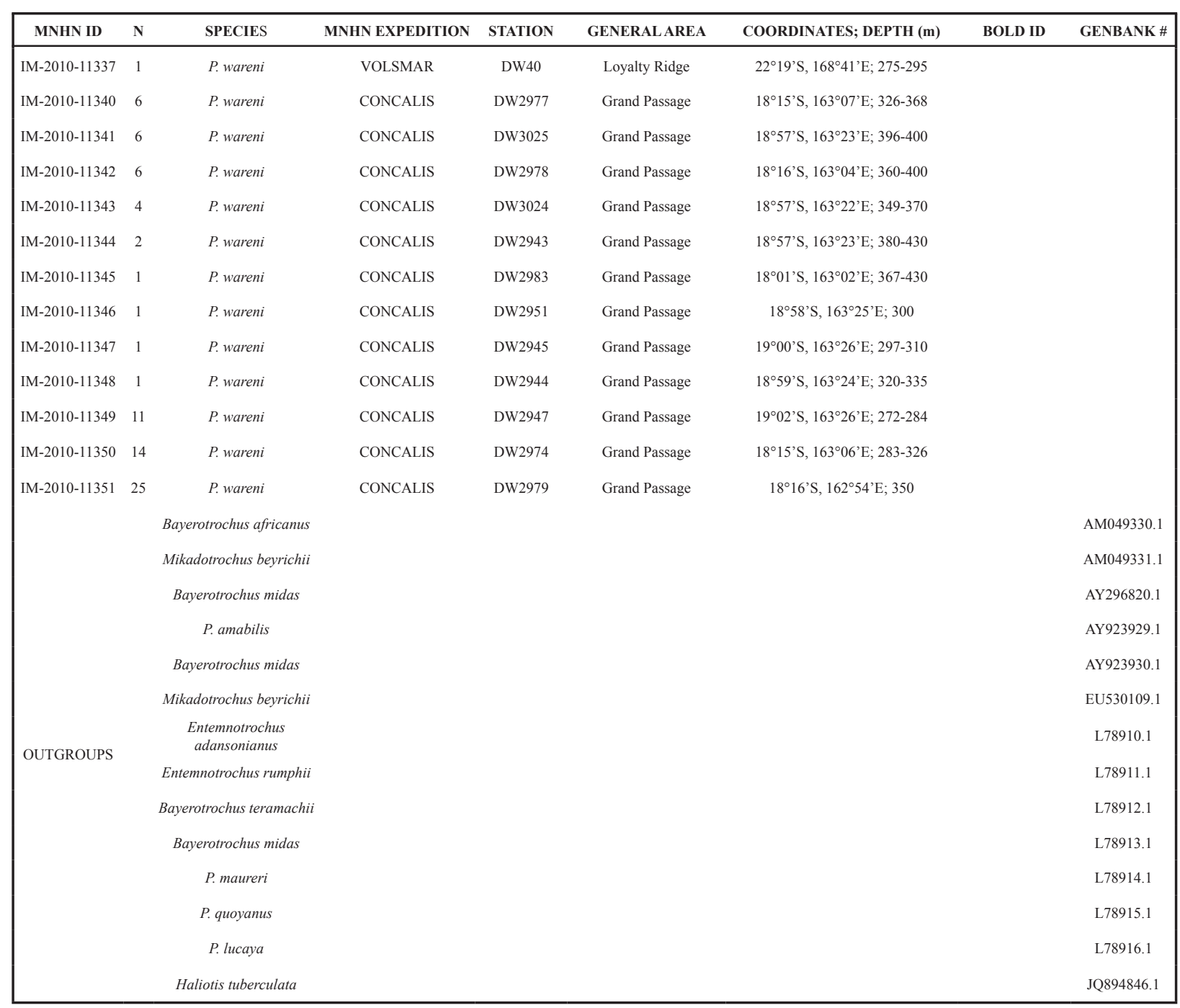


Table 2. Shell characters in the Perotrochus caledonicus species complex. Measurements correspond to mean values (with ranges) calculated on $\mathrm{N}$ specimens. Values for $P$. deforgesi are taken from Métivier (1990).

\begin{tabular}{|c|c|c|c|c|}
\hline Character & Perotrochus caledonicus & $\begin{array}{l}\text { Perotrochus wareni sp. } \\
\text { nov. }\end{array}$ & $\begin{array}{c}\text { Perotrochus } \\
\text { pseudogranulosus sp. nov. }\end{array}$ & $\begin{array}{l}\text { Perotrochus } \\
\text { deforgesi }\end{array}$ \\
\hline General appearance & $\begin{array}{l}\text { trochoid with regularly } \\
\text { increasing weakly convex } \\
\text { whorls }\end{array}$ & $\begin{array}{l}\text { conical, with weakly } \\
\text { convex to rather } \\
\text { straight-sided whorls }\end{array}$ & $\begin{array}{c}\text { rather conical, with weakly } \\
\text { convex to straight-sided } \\
\text { whorls }\end{array}$ & depressed turbiniform \\
\hline $\mathrm{H} / \mathrm{D}$ ratio & $1.00(0.92-1.00, \mathrm{~N}=7)$ & $1.05(1.01-1.09, \mathrm{~N}=6)$ & $0.87(0.84-0.92, \mathrm{~N}=9)$ & $0.76(\mathrm{~N}=20)$ \\
\hline $\begin{array}{l}\text { Shell weight / } \\
\text { thickness }\end{array}$ & light, thin shell & very heavy, thick shell & light, thin shell & thick, heavy shell \\
\hline Apical whorls, profile & $\begin{array}{l}\text { acute sharp / straight conical } \\
\text { (with no whitish cord } \\
\text { protruding on surface) }\end{array}$ & $\begin{array}{c}\text { more depressed, with } \\
\text { whitish cord protruding } \\
\text { near suture of apical } \\
\text { whorls }\end{array}$ & straight conical & depressed \\
\hline $\begin{array}{l}\text { Dominant teleoconch } \\
\text { sculpture }\end{array}$ & $\begin{array}{l}\text { macroscopically generally } \\
\text { unbeaded to slightly beaded, } \\
\text { straight spiral cords }\end{array}$ & $\begin{array}{c}\text { macroscopically finely } \\
\text { but distinctly beaded } \\
\text { spiral cords, variable } \\
\text { strength with crenulated } \\
\text { periphery }\end{array}$ & $\begin{array}{l}\text { distinct finely light beaded } \\
\text { spiral cords, with angled } \\
\text { periphery }\end{array}$ & $\begin{array}{c}\text { strongly beaded spira } \\
\text { ribs above and under } \\
\text { selenizone, with } \\
\text { angled periphery }\end{array}$ \\
\hline \multirow{2}{*}{$\begin{array}{l}\text { Teleoconch } \\
\text { microsculpture }\end{array}$} & \multirow{2}{*}{$\begin{array}{c}\text { fine diverging radiating } \\
\text { threads }\end{array}$} & \multirow{2}{*}{$\begin{array}{l}\text { no diverging radiating } \\
\text { microsculpture }\end{array}$} & fine microsculpture & microsculpture \\
\hline & & & radiating threads & radiating threads \\
\hline $\begin{array}{l}\text { Nacreous coverage } \\
\text { of inner slit lips at } \\
\text { aperture edge }\end{array}$ & $\begin{array}{c}\text { nearly completely covered } \\
\text { by nacre }(90 \%) \text {, small area } \\
\text { with porcellaneous layer } \\
\text { parallel to slit margins }\end{array}$ & $\begin{array}{c}\text { partially covered by } \\
\text { nacre }(75 \%) \text {, leaving } \\
\text { V-shaped area with } \\
\text { porcellaneous layer } \\
\text { uncovered }(25 \%) \\
\end{array}$ & $\begin{array}{l}\text { nearly completely covered } \\
\text { by nacre }(85 \%) \text {, small area } \\
\text { with porcellaneous layer } \\
\text { parallel to slit margins }\end{array}$ & $\begin{array}{c}\text { partially covered } \\
\text { by nacre }(80 \%), \\
\text { porcellaneous } \\
\text { layer parallel to slit } \\
\text { uncovered } \\
\end{array}$ \\
\hline Slit width & wide & very narrow & rather wide & rather wide \\
\hline Slit length & $\begin{array}{c}\text { long, } 1 / 6^{\text {th }} \text { basal diameter } \\
(1 / 5.7-1 / 6.6, N=7)\end{array}$ & $\begin{array}{c}\text { shorter, } 1 / 7^{\text {th }} \text { basal } \\
\text { diameter }(1 / 6.5-1 / 7.6 \\
\mathrm{N}=6)\end{array}$ & $\begin{array}{c}\text { long, about } 1 / 5^{\text {th }} \text { basal } \\
\text { diameter }(1 / 5.4-1 / 5.8, \\
\mathrm{N}=8)\end{array}$ & $\begin{array}{l}\text { long, } 1 / 5^{\text {th }} \text { basal } \\
\text { diameter }(\mathrm{N}=7)\end{array}$ \\
\hline Whorl surface & dull & dull & metallic lustre & slightly lustruous \\
\hline $\begin{array}{c}\text { Checker-board colour } \\
\text { pattern in areas below } \\
\text { selenizone }\end{array}$ & $\begin{array}{l}\text { not always clearly marked; } \\
\text { rather weak colour intensity, } \\
\text { not limited to the area } \\
\text { below selenizone }\end{array}$ & $\begin{array}{l}\text { more irregularly } \\
\text { sized and spaced } \\
\text { colour blocks of less } \\
\text { contrasting intensity }\end{array}$ & $\begin{array}{l}\text { very regularly spaced } \\
\text { checkerboard color } \\
\text { markings of deeper colour } \\
\text { intensity; overall deeper } \\
\text { colour of teleoconch }\end{array}$ & $\begin{array}{l}\text { orange blotches of } \\
\text { variable intensity } \\
\text { in areas above } \\
\text { selenizone, not below }\end{array}$ \\
\hline $\begin{array}{c}\text { Surface of umbilical } \\
\text { callus pad on basal } \\
\text { disc }\end{array}$ & $\begin{array}{c}\text { medium large } \\
(34 \%-29.1-35.2, \\
\mathrm{N}=6) \text {, generally smooth } \\
\text { or weakly ridged radially in } \\
\text { large adults } \\
\end{array}$ & $\begin{array}{l}\text { very large }(45 \% \text { - } \\
38.0-48.9, \mathrm{~N}=6) \text {, ridged } \\
\text { radially, sharply edged } \\
\text { at margin }\end{array}$ & $\begin{array}{c}\text { small }(29 \%-19.6-40.5 \\
\mathrm{N}=9), \text { finely ridged } \\
\text { radially, with clearly raised } \\
\text { edge }\end{array}$ & $\begin{array}{l}\text { large }(36 \%-\mathrm{N}=7) \text {, } \\
\text { clearly ridged radially } \\
\text { and with raised edge }\end{array}$ \\
\hline Basal disc profile & generally rather convex & flattened & rather flattened & rather flattened \\
\hline
\end{tabular}

